\title{
Chicken bromodomain-containing protein 2 interacts with the Newcastle disease virus matrix protein and promotes viral replication
}

\author{
Zhiqiang Duan ${ }^{1,2^{*}} \mathbb{0}$, Yifan Han², Lei Zhou², Chao Yuan², Yanbi Wang ${ }^{2}$, Caiqin Zhao², Hong Tang ${ }^{2}$ \\ and Jiaqi Chen ${ }^{2}$
}

\begin{abstract}
Bromodomain-containing protein 2 (BRD2) is a nucleus-localized serine-threonine kinase that plays pivotal roles in the transcriptional control of diverse genes. In our previous study, the chicken BRD2 (chBRD2) protein was found to interact with the Newcastle disease virus (NDV) matrix (M) protein using a yeast two-hybrid screening system, but the role of the chBRD2 protein in the replication of NDV remains unclear. In this study, we first confirmed the interaction between the M protein and chBRD2 protein using fluorescence co-localization, co-immunoprecipitation and pull-down assays. Intracellular binding studies indicated that the C-terminus (aa 264-313) of the M protein and the extra-terminal (ET) domain (aa 619-683) of the chBRD2 protein were responsible for interactions with each other. Interestingly, although two amino acids (T621 and S649) found in the chBRD2/ET domain were different from those in the human BRD2/ET domain and in that of other mammals, they did not disrupt the BRD2-M interaction or the chBRD2-M interaction. In addition, we found that the transcription of the chBRD2 gene was obviously decreased in both NDV-infected cells and pEGFP-M-transfected cells in a dose-dependent manner. Moreover, small interfering RNA-mediated knockdown of chBRD2 or overexpression of chBRD2 remarkably enhanced or reduced NDV replication by upregulating or downregulating viral RNA synthesis and transcription, respectively. Overall, we demonstrate for the first time that the interaction of the M protein with the chBRD2 protein in the nucleus promotes NDV replication by downregulating chBRD2 expression and facilitating viral RNA synthesis and transcription. These results will provide further insight into the biological functions of the M protein in the replication of NDV.
\end{abstract}

Keywords: Newcastle disease virus, matrix protein, bromodomain-containing protein 2, viral replication

\section{Introduction}

Newcastle disease (ND) is an important avian infectious viral disease that causes neurological, respiratory, and gastrointestinal symptoms in poultry and may lead to devastating losses in the poultry industry worldwide [1]. The causative agent of ND is Newcastle disease virus (NDV), also known as avian paramyxovirus type 1 , which

\footnotetext{
*Correspondence: zqduan@gzu.edu.cn

${ }^{1}$ Key Laboratory of Animal Genetics, Breeding and Reproduction

in the Plateau Mountainous Region, Ministry of Education, Guizhou University, Guiyang, China

Full list of author information is available at the end of the article
}

belongs to the genus Orthoavulavirus of the subfamily Paramyxoviridae [2]. The genome of NDV is a nonsegmented, negative-sense, single-stranded RNA that encodes eight proteins, including six structural proteins [the nucleocapsid protein (NP), phosphoprotein protein $(\mathrm{P})$, matrix protein $(\mathrm{M})$, fusion protein $(\mathrm{F})$, haemagglutinin-neuraminidase protein $(\mathrm{HN})$ and large polymerase protein $(\mathrm{L})]$ as well as two non-structural proteins $(\mathrm{V}$ and $\mathrm{W})$ derived from RNA editing of the P gene $[3,4]$. Of all these viral structural proteins, the M protein is surrounded by the inner surface of the viral envelope and forms an outer protein shell around the nucleocapsid, which constitutes the bridge between the viral envelope

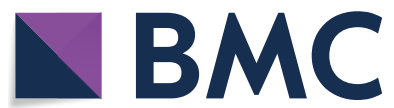

(c) The Author(s) 2020. This article is licensed under a Creative Commons Attribution 4.0 International License, which permits use, sharing, adaptation, distribution and reproduction in any medium or format, as long as you give appropriate credit to the original author(s) and the source, provide a link to the Creative Commons licence, and indicate if changes were made. The images or other third party material in this article are included in the article's Creative Commons licence, unless indicated otherwise in a credit line to the material. If material is not included in the article's Creative Commons licence and your intended use is not permitted by statutory regulation or exceeds the permitted use, you will need to obtain permission directly from the copyright holder. To view a copy of this licence, visit http://creativeco mmons.org/licenses/by/4.0/. The Creative Commons Public Domain Dedication waiver (http://creativecommons.org/publicdomain/ zero/1.0/) applies to the data made available in this article, unless otherwise stated in a credit line to the data. 
and the nucleocapsid [5]. Like the $M$ protein of most paramyxoviruses, the NDV M protein is also a multifunctional nucleocytoplasmic trafficking protein [6]. In addition to participating in assembly in the cytoplasm and the budding of progeny virions at the cell membrane later in infection [7], the NDV M protein is localized in the nucleus and nucleolus early in infection $[8,9]$, which may inhibit host cell transcription and protein synthesis [10]. Recent studies have shown that nuclear-cytoplasmic trafficking of the NDV M protein is mediated by its intrinsic nuclear localization signal (NLS) and nuclear export signals (NESs) [11, 12]. Additionally, M/NLS mutation results in a pathotype change of NDV and attenuates viral replication and pathogenicity [11], while M/NES mutation causes ineffective rescue of NDV [12], demonstrating that nucleocytoplasmic trafficking of the NDV M protein plays crucial roles in the virus life cycle.

In recent years, an increasing number of studies have focused on mutation of some amino acids in the NDV M protein, such as the $\mathrm{N}$-terminal ${ }^{23} \mathrm{FPIV}^{26}$ motif [13], basic amino acids R36 [14] and R42 [15], and C-terminal G275 and P276 [16], to understand its functions. Additionally, exploration of the interaction of the $M$ protein with cellular proteins has also been used to study the roles of the $M$ protein in the replication and pathogenesis of NDV. For example, the NDV M protein interacting with the human Bax protein is beneficial to elucidate the proapoptotic ability of NDV [17]. In addition, host charged multivesicular body protein $4 \mathrm{~B}$ or nucleophosmin interacts with the NDV M protein, which is essential for the replication of NDV and the nucleolar targeting of the M protein, respectively $[18,19]$. Importantly, a recent study found that the interaction of the NDV M protein with the antiviral protein viperin reduces virus replication, suggesting for the first time that the $\mathrm{M}$ protein is involved in NDV immune evasion [20]. In our recent studies, several cellular proteins were found to interact with the NDV $M$ protein using a yeast two-hybrid screening system, of which the chicken transcriptional regulatory factor bromodomain-containing protein 2 (BRD2) was a novel M-interacting partner that may regulate the replication of NDV [11], but the precise function of this interaction in NDV replication remains unclear.

BRD2 belongs to the bromodomain and extra-terminal domain (BET) family and contains two tandem bromodomains (BD1 and BD2) and an extra-terminal (ET) domain, of which the bromodomain is required for the epigenetic regulation of gene transcription by BET proteins through interacting with nucleosomes within chromatin, and the ET domain fulfils its regulatory functions by recruiting specific effector proteins [21, 22]. BRD2 is reported to be a nuclear transcription factor kinase and acts as a transcriptional regulator with switch mating type/sucrose non-fermenting (SWI/SNF)-like functions that regulate chromatin remodelling, inflammatory responses, cell-cycle progression and so on [23-25]. More importantly, BRD2 can also bind transcriptional activators, such as E2F proteins, and co-activators, including TATA-binding protein (TBP)-associated factors (TAFs), histone acetyltransferases and histone deacetylases, to regulate the transcription of diverse genes [26]. The NDV M protein is localized in the nucleus early in infection and does not require other NDV proteins $[8$, 9]. However, which cellular proteins in the nucleus the NDV M protein interacts with and how these interactions regulate virus replication remain unknown. In this study, the role of chicken BRD2 (chBRD2) in the life cycle of NDV was investigated. We demonstrated for the first time that the NDV M protein directly interacts with the chBRD2 protein in the nucleus, which downregulates the expression of chBRD2 and facilitates viral RNA synthesis and transcription to promote NDV replication.

\section{Materials and methods \\ Cells, viruses and antibodies}

Chicken embryonic fibroblasts (DF-1) and human embryonic kidney 293T (HEK-293T) cells were purchased from the Cell Resource Center of Shanghai Institutes for Biological Sciences of the Chinese Academy of Sciences. The NDV strain expressing green fluorescent protein (GFP) (rSS1GFP) was generated in our previous study [11]. The virus was plaque purified three times in DF-1 cells and propagated once in specific pathogen-free (SPF) embryonated chicken eggs. Primary mouse antiHA tag monoclonal antibody (T0008), mouse anti-Myc tag monoclonal antibody (T0001), rabbit anti-Myc tag polyclonal antibody (T0052), mouse anti-His tag monoclonal antibody (T0009), mouse anti-GST tag monoclonal antibody (T0007), mouse anti-GFP tag monoclonal antibody (T0005), mouse anti-GAPDH monoclonal antibody (T0004), Alexa Fluor 488-conjugated goat antimouse IgG ( $\mathrm{H}+\mathrm{L})$ antibody (S0017) and Cy3-conjugated goat anti-rabbit IgG $(\mathrm{H}+\mathrm{L})$ antibody (S0011) were purchased from Affinity Biosciences (USA). Rabbit antiNDV M polyclonal antibody was prepared by Wuhan GeneCreate Biological Engineering Co., Ltd (China).

\section{Plasmid construction}

The plasmids pEGFP-C1, pDsRed-C1, pCMV-HA, and pCMV-Myc were purchased from Clontech (USA), and $\mathrm{pET}-32 \mathrm{a}(+)$ and pGEX-6p-1 were purchased from Novagen (USA) and GE Healthcare (USA), respectively. All enzymes used for cloning procedures were purchased from Thermo Fisher Scientific Company (USA). The open reading frame (ORF) of the $M$ gene (GenBank No. KP742770) was amplified from the plasmid 
pNDV/SS1GFP [11] and then subcloned into the plasmids pEGFP-C1, pCMV-HA, and pGEX-6p-1 to generate pEGFP-C1-M, pCMV-HA-M and pGEX-6p-M, respectively. The ORF of the chBRD2 gene (GenBank No. XM_015294960) was amplified from cDNA derived from DF-1 cells and then used to construct the recombinant expression plasmids pDsRed-chBRD2, pCMV-MycchBRD2, and pET-32a-chBRD2. Plasmids expressing the truncation mutants or deletion mutants of the $M$ and chBRD2 proteins were constructed by inserting PCRgenerated fragments into the plasmid pEGFP-C1, pCMVHA or pCMV-Myc. The gene fragments of chBRD2/ ET and human BRD2/ET (huBRD2/ET) (GenBank No. NM_005104) were amplified from cDNA derived from DF-1 cells and HEK-293T cells, respectively, and then subcloned into the plasmid pCMV-Myc to generate pCMV-Myc-chBRD2/ET and pCMV-Myc-huBRD2/ET. Primers used for the construction of the above recombinant expression plasmids are shown in Table 1. All of the constructed recombinant expression plasmids were confirmed by PCR, restriction digestion and DNA sequencing.
Cell culture, transfection and fluorescence microscopy

DF-1 cells were maintained in Dulbecco's modified Eagle medium (Gibco, USA) containing 12\% foetal bovine serum (FBS) (Gibco, USA) at $37^{\circ} \mathrm{C}$ under an atmosphere with $5 \% \mathrm{CO}_{2}$. For the transfection experiments, $5 \times 10^{5}$ DF-1 cells were grown to $80 \%$ confluence in 6-well plates and then double (pEGFP-M and pDsRed-chBRD2 or pCMV-HA-M and pCMV-Myc-chBRD2) transfected with a total of $3 \mu \mathrm{g}$ of plasmid ( $1.5 \mu \mathrm{g}$ for each plasmid) using FuGENE HD Transfection Reagent (Promega, USA) according to the manufacturer's instructions. Twenty-four hours after transfection, DF-1 cells expressing the recombinant proteins EGFP-M and DsRedchBRD2 were rinsed with phosphate-buffered saline (PBS), fixed with pre-cooled $4 \%$ paraformaldehyde for $20 \mathrm{~min}$ at room temperature, permeabilized with $0.25 \%$ Triton X-100 for $5 \mathrm{~min}$, and then counterstained with DAPI (Sigma, USA) to detect nuclei. For the fluorescence detection of the recombinant proteins HA-M and MycchBRD2, plasmid-co-transfected DF-1 cells were rinsed, fixed, permeabilized as described above, blocked with $10 \% \mathrm{FBS}$ in PBS for $1 \mathrm{~h}$, and then incubated with mouse

Table 1 Primers used for the construction of recombinant expression plasmids

\begin{tabular}{|c|c|c|c|}
\hline Recombinant plasmid & Sense primer $\left(5^{\prime} \rightarrow 3^{\prime}\right)$ & Anti-sense primer $\left(5^{\prime} \rightarrow 3^{\prime}\right)$ & Restriction sites ${ }^{\mathrm{a}}$ \\
\hline pEGFP-M & TCGGAATTCAATGGACTCATCCAGGAC & GCAGTCGACTTATTTCCTGAAAGG & EcoRI/Sall \\
\hline pCMV-HA-M & TTGAATTCGGATGGACTCATCCAGGAC & GCACTCGAGTTATTTCCTGAAAGG & EcoRl/Xhol \\
\hline pGEX-6p-M & TGCGAATTCATGGACTCATCCAGGAC & GGCACTCGAGTTATTTCCTGAAAGG & EcoRl/Xhol \\
\hline pDsRed-chBRD2 & CAGCTCGAGCTATGCTGCAGAATGTGAATCC & TGGGATCCTTAACCCGAATCCGAGTCGCT & Xhol/BamHI \\
\hline pCMV-Myc-chBRD2 & AGGAATTCAAATGCTGCAGAATGTGAATCC & TGCTCGAGTTAACCCGAATCCGAGTCGC & EcoRl/Xhol \\
\hline pET-32a-chBRD2 & CAGGAATTCATGCTGCAGAATGTGAATCC & TGCTCGAGTTAACCCGAATCCGAGTCGC & EcoRl/Xhol \\
\hline pCMV-HA-M(1-178) & TTGAATTCGGATGGACTCATCCAGGAC & GACTCGAGTTACAAGGAGACAAAATTCAC & EcoRl/Xhol \\
\hline pCMV-HA-M(1-245) & TTGAATTCGGATGGACTCATCCAGGAC & AACTCGAGTTATACAGTGGACATAAGCCC & EcoRl/Xhol \\
\hline pCMV-HA-M(1-263) & TTGAATTCGGATGGACTCATCCAGGAC & GACTCGAGTTATCTCCTTATCTTTTCCTC & EcoRl/Xhol \\
\hline pCMV-HA-M(264-364) & GAGAATTCGGCTCAATCTATCTGTTGG & GCACTCGAGTTATTTCCTGAAAGG & EcoRl/Xhol \\
\hline pCMV-HA-M(314-364) & GAGAATTCGGATACTCTGGAGCCAGACC & GCACTCGAGTTATTTCCTGAAAGG & EcoRl/Xhol \\
\hline pCMV-HA-M(264-313) & GAGAATTCGGCTCAATCTATCTGTTGGG & CTCTCGAGTTACTTGGCAACCTGGGGAGA & EcoRl/Xhol \\
\hline pCMV-Myc-chBRD2(1-179) & AGGAATTCAAATGCTGCAGAATGTGAATCC & TCCTCGAGTTACATTTGGGCCACCTTCTGCA & EcoRl/Xhol \\
\hline pCMV-Myc-chBRD2(1-447) & AGGAATTCAAATGCTGCAGAATGTGAATCC & GGCTCGAGTTAGTAGCTGAACTCAAAGACATCC & EcoRl/Xhol \\
\hline pCMV-Myc-chBRD2(1-510) & AGGAATTCAAATGCTGCAGAATGTGAATCC & TCCTCGAGTTAGTCAGAGCTCTCCTCGCTCTCT & EcoRl/Xhol \\
\hline pCMV-Myc-chBRD2(511-779) & GAGAATTCTCTCGGAAGAGGAACGTGCCAACC & TGCTCGAGTTAACCCGAATCCGAGTCGC & EcoRl/Xhol \\
\hline pCMV-Myc-chBRD2(619-683) & AGGAATTCAACCGATGACGTACGATGAGAAG & TTCTCGAGTTAGGACAGCACGTAGCGCTCCAG & EcoRl/Xhol \\
\hline pCMV-Myc-chBRD2/ET & AGGAATTCAACCGATGACGTACGATGAGAAG & TTCTCGAGTTAGGACAGCACGTAGCGCTCCAG & EcoRl/Xhol \\
\hline pCMV-Myc-huBRD2/ET & AGGAATTCAACCCATGAGTTACGATGAGAAG & TTCTCGAGTTAGGACAGCACGTAGCGCTCCAG & EcoRl/Xhol \\
\hline \multirow[t]{2}{*}{ pCMV-HA-M( $(\triangle 264-313)$} & TTGAATTCGGATGGACTCATCCAGGAC & CTGGCTCCAGAGTATTCTCCTTATCTTTTCCTC & EcoRl \\
\hline & GAAAAGATAAGGAGAATACTCTGGAGCCAGACC & GCACTCGAGTTATTTCCTGAAAGG & Xhol \\
\hline \multirow[t]{2}{*}{ pEGFP-M( $\triangle 264-313)$} & TCGGAATTCAATGGACTCATCCAGGAC & CTGGCTCCAGAGTATTCTCCTTATCTTTTCCTC & ECORI \\
\hline & GAAAAGATAAGGAGAATACTCTGGAGCCAGACC & GCAGTCGACTTATTTCCTGAAAGG & Sall \\
\hline \multirow[t]{2}{*}{ pCMV-Myc-chBRD2( $\triangle 619-683)$} & AGGAATTCAAATGCTGCAGAATGTGAATCC & TTTCTTCCGCAGGCATTTGCTCTCCTCTTCCTC & EcoRl \\
\hline & GAAGAGGAGAGCAAATGCCTGCGGAAGAAACCC & TGCTCGAGTTAACCCGAATCCGAGTCGC & Xhol \\
\hline
\end{tabular}

\footnotetext{
${ }^{a}$ Restriction sites are given in italics and underlined.
} 
anti-HA and rabbit anti-Myc antibodies for $1 \mathrm{~h}$. After three washes with PBS, the cells were incubated with Alexa Fluor 488-conjugated goat anti-mouse IgG $(\mathrm{H}+\mathrm{L})$ and $\mathrm{Cy3}$-conjugated goat anti-rabbit IgG $(\mathrm{H}+\mathrm{L})$ antibodies for $1 \mathrm{~h}$. Cells were also counterstained with DAPI to detect nuclei. Fluorescent images were obtained under an inverted fluorescence microscope and then analysed and merged with Adobe Photoshop CS7 software.

\section{Protein interaction assays}

For co-immunoprecipitation (co-IP) assays, $5 \times 10^{5}$ DF- 1 cells cultured in 6-well plates were co-transfected with the indicated plasmids co-expressing the full-length $M$ protein and chBRD2 protein or their truncation mutants. At $36 \mathrm{~h}$ post-transfection (hpt), cells were washed three times with PBS and then lysed with immunoprecipitation lysis buffer (Pierce, USA). The supernatants were collected after centrifugation and then incubated with an anti-HA or anti-Myc antibody overnight at $4{ }^{\circ} \mathrm{C}$. The immune complexes were recovered by adsorption to protein A+ G-Sepharose (Sigma, USA) for $3 \mathrm{~h}$ at $4{ }^{\circ} \mathrm{C}$. After three washes with immunoprecipitation lysis buffer, the immunoprecipitates were detected by western blotting using an anti-Myc or anti-HA antibody.

For pull-down assays, the His-chBRD2 fusion protein $\left(4 \mathrm{~h}\right.$ of induction with $0.5 \mathrm{mM}$ IPTG at $\left.25^{\circ} \mathrm{C}\right)$ and GST-M fusion protein ( $4 \mathrm{~h}$ of induction with $0.5 \mathrm{mM}$ IPTG at $28{ }^{\circ} \mathrm{C}$ ) were expressed in E. coli BL21 (DE3), and soluble His-chBRD2 and GST-M were purified on Ni-NTA His*Bind Resin (Merck, USA) and GlutathioneSepharose 4B beads (GE Healthcare, USA), respectively. In the GST pull-down assay, the purified GST-M protein was immobilized on Glutathione-Sepharose 4B beads. After washing with transport buffer, the immobilized protein was incubated with purified His-chBRD2 for $2 \mathrm{~h}$ at $4{ }^{\circ} \mathrm{C}$. The beads were then washed three times with transport buffer, and the target protein His-chBRD2 was eluted from the beads and used for SDS-PAGE followed by western blot analysis. In the His pull-down assay, His*Bind Resin-bound His-chBRD2 was incubated with purified GST-M for $2 \mathrm{~h}$ at $4{ }^{\circ} \mathrm{C}$. The resins were treated as described above, and the target protein GST-M was detected by western blotting.

\section{Multiple sequence alignment and computer modelling}

The complete coding sequences of the BRD2 gene from humans and other selected species were retrieved from the GenBank database. The amino acid sequences of the BRD2 protein (huBRD2 as a template) were analysed using the Clustal W multiple alignment algorithm in the MegAlign program of the DNASTAR Lasergene package, version 7.1 (DNASTAR Inc. Madison, WI, USA). The amino acid conservation of the BRD2/ET domain among humans and different species was obtained based on the above multiple sequence alignment results. The three-dimensional crystal structure of the huBRD2/ET domain (Protein Data Bank [PDB] accession no. 6CUI) was used as a template to illustrate the structural difference between the huBRD2/ET domain and chBRD2/ET domain. This modelling was performed with PyMOL software (Schrödinger).

\section{The effect of the NDV M protein on the transcription of the chBRD2 gene}

To study the effect of the NDV M protein on the transcription of the chBRD2 gene, $5 \times 10^{5}$ low-passage DF-1 cells grown to $80 \%$ confluence in 6 -well plates were infected with rSS1GFP at an MOI of $0.01,0.1$ or 1 , or transfected with the plasmid pEGFP-M, pEGFP$\mathrm{M}(\Delta 264-313)$ ( $\mathrm{M}$ protein deleting residues 264 to 313 ), or pEGFP-C1 at a dose of $1.0 \mu \mathrm{g}, 2.0 \mu \mathrm{g}$ or $4.0 \mu \mathrm{g}$. The expression levels of the $M$ protein in rSS1GFP-infected DF-1 cells were detected at 6,12 and $24 \mathrm{hpi}$, while the expression levels of EGFP-M, EGFP-M $(\Delta 264-313)$ and EGFP in DF-1 cells transfected with different doses of plasmid were examined at $36 \mathrm{hpt}$. The relative levels of the selected proteins to those of the control GAPDH were determined by densitometry using ImageJ software version 1.8.0. In addition, quantitative real-time PCR (qRT-PCR) was used to detect the transcription of the endogenous chBRD2 gene in virus-infected or plasmidtransfected DF-1 cells using a SYBR Premix Ex Taq Kit (Takara Biomedical Technology, China) according to the manufacturer's instructions. All the reactions were performed in a $10 \mu \mathrm{L}$ volume containing $5 \mu \mathrm{L}$ of $2 \times$ SYBR Premix Ex Taq, $200 \mathrm{nM}$ each primer, and $0.2 \mu \mathrm{L}$ of ROX reference Dye II. The cycling parameters were 1 cycle at $95{ }^{\circ} \mathrm{C}$ for $5 \mathrm{~s}$, followed by 40 cycles at $95^{\circ} \mathrm{C}$ for $5 \mathrm{~s}$ and $60{ }^{\circ} \mathrm{C}$ for $31 \mathrm{~s}$. One cycle of melting curve analysis was added for all reactions to verify product specificity. The relative transcription levels of the $\operatorname{ch} B R D 2$ gene were normalized to those of the GAPDH gene. The threshold cycle $2^{-\Delta \Delta C T}$ method was used to determine the fold change in gene transcription levels.

\section{Small interfering RNA (siRNA) treatment and virus infection}

The sequences of three pairs of siRNAs designed to knockdown the chBRD2 gene (GenBank No. XM_015294960) in DF-1 cells are shown in Table 2. Negative control siRNA (Cat. No. 12935-400) and siRNA transfection reagent Lipofectamine ${ }^{\mathrm{TM}}$ RNAiMAX were purchased from Thermo Fisher Scientific Company (USA). For transfection with the siRNA against chBRD2, low-passage DF-1 cells grown to $80 \%$ confluence in 6-well plates were transfected with the indicated siRNAs 
Table 2 Information on chBRD2 siRNAs

\begin{tabular}{|c|c|c|c|}
\hline Name & siRNA Name & Sequence $\left(5^{\prime} \rightarrow 3^{\prime}\right)$ & \\
\hline \multirow[t]{6}{*}{ chBRD2 (GenBank no. XM_015294960) } & SiRNA\#1 & Sense & CAAACUGCUAUAUCUAUAACA \\
\hline & & Anti-sense & UUAUAGAUAUAGCAGUUUGUG \\
\hline & siRNA\#2 & Sense & ACUGCUAUAUCUAUAACAAGC \\
\hline & & Anti-sense & UUGUUAUAGAUAUAGCAGUUU \\
\hline & siRNA\#3 & Sense & GCUCCAAGAACUCAAAGAAAG \\
\hline & & Anti-sense & UUCUUUGAGUUCUUGGAGCUG \\
\hline \multirow[t]{2}{*}{ Control } & Negative siRNA & Sense & UUCUCCGAACGUGUCACGUTT \\
\hline & & Anti-sense & ACGUGACACGUUCGGAGAATT \\
\hline
\end{tabular}

$(10 \mu \mathrm{M})$ at a dose of $30 \mathrm{pmol}$, and the knockdown efficiency was detected by qRT-PCR at $48 \mathrm{hpt}$, as described above. To study the effect of chBRD2 knockdown on the replication of NDV, at $48 \mathrm{hpt}$, rSS1GFP was used to infect chBRD2 siRNA\#2- or control siRNA-treated DF-1 cells or non-transfected cells at an MOI of 1 . The cell culture supernatants were collected at the indicated time points $(6,12,24,36,48$, and $72 \mathrm{hpi})$, and the virus titres were titrated using $50 \%$ tissue culture infective doses $\left(\mathrm{TCID}_{50}\right)$ in DF-1 cells according to the Reed and Muench method [27]. Meanwhile, the cytopathic effect (CPE) in virusinfected cells was observed under an inverted fluorescence microscope, and the expression of the $M$ protein and GFP protein was examined by western blotting at 6 , 12 and 24 hpi. The relative levels of the selected proteins compared to control GAPDH expression were determined by densitometry using Image J software version 1.8.0.

\section{chBRD2 overexpression and virus infection}

To further investigate the effect of chBRD2 overexpression on the replication of NDV, low-passage DF-1 cells at $80 \%$ confluence in 6-well plates were transfected with $3.0 \mu \mathrm{g}$ of pDsRed-chBRD2 or pDsRed-C1 to overexpress DsRed-chBRD2 or DsRed. The transfection efficiency and transcription level of pDsRed-chBRD2 were examined by fluorescence observation and qRT-PCR at $36 \mathrm{hpt}$, respectively, and then the plasmid-transfected DF-1 cells were infected with rSS1GFGP at an MOI of 1 . The cell culture supernatants were collected at the indicated time points $(6,12,24,36,48$, and $72 \mathrm{hpi})$, and the virus titres were determined in DF-1 cells. In addition, the expression levels of the M protein and GFP protein in DsRedoverexpressing cells, DsRed-chBRD2-overexpressing cells and non-transfected cells were examined by western blotting at 6,12 and 24 hpi. The relative levels of the selected proteins compared to control GAPDH expression were determined by densitometry using ImageJ software version 1.8.0.

\section{Quantification of viral RNA synthesis and gene transcription by qRT-PCR}

The primers used for the quantification of viral RNA synthesis and gene expression by qRT-PCR were designed based on the target sequences using Primer Premier 5.0 software (Table 3). siRNA- or plasmid-transfected DF-1 cells were collected from virus infection assays at the indicated time points $(6,12$ and $24 \mathrm{hpi})$, and total RNA was extracted using TRIzol reagent (Invitrogen, USA) according to the manufacturer's protocol. One microgram of total RNA per sample was reverse-transcribed using a previously described method $[28,29]$. Briefly, the primer PRT-G (5'-ACGATAAAAGGCGGAGAAGCA$3^{\prime}$, nucleotide positions $24-44$ in the SS1 genome) specific for negative-sense viral RNA was used for reverse transcription. Reverse transcripts were stored at $-80^{\circ} \mathrm{C}$ until use in qRT-PCR assays. The primers qNDV/NP-F and $\mathrm{qNDV} / \mathrm{NP}-\mathrm{R}$ and $\mathrm{qNDV} / \mathrm{P}-\mathrm{F}$ and $\mathrm{qNDV} / \mathrm{P}-\mathrm{R}$ were used to quantify the viral $N P$ gene and $P$ gene, respectively. On the other hand, $1 \mu \mathrm{g}$ of total RNA per sample was reverse-transcribed into cDNA using Superscript IV reverse transcriptase (Invitrogen, USA). The primers qNDV/M-F and qNDV/M-R and qGFP-F and qGFP-R were used to quantify the viral $M$ gene and GFP gene, respectively. qRT-PCR experiments were performed using a SYBR Premix Ex Taq Kit (Takara Biomedical Technology, China). The qRT-PCR procedure used to quantify viral RNA synthesis and gene transcription was carried out according to our previously described method [10]. The relative gene transcription levels were normalized to that of the GAPDH gene. The threshold cycle $2^{-\Delta \Delta \mathrm{CT}}$ method was used to determine the fold change in gene transcription levels.

\section{Statistical analysis}

Differences in the expression levels of genes and proteins and virus titres were analysed using SPSS 12.0 software (SPSS Inc., USA). An independent-samples $t$ test was used for data analysis. All experiments were repeated 
Table 3 Quantitative real-time PCR primers used in this study

\begin{tabular}{|c|c|c|c|}
\hline Primer name & Sequence $\left(5^{\prime} \rightarrow 3^{\prime}\right)$ & Product size (bp) & GenBank no. \\
\hline qchBRD2-F & AAAGTGGTGATGAAAGCCCTGTGGA & 113 & XM_015294960 \\
\hline qchBRD2-R & ATGGGCTGCTTGATGATCTTGTGGT & & \\
\hline qNDV/NP-F & TTACAACTTGGTCGGGGATGTAGAC & 166 & KP742770 \\
\hline qNDV/NP-R & CATCCGATATAAACGCATGAGCTG & & \\
\hline qNDV/P-F & TATGGAAGCAACCAGGGAAGACC & 185 & KP742770 \\
\hline qNDV/P-R & TGGACACGATCCACAGGTACAGGA & & \\
\hline qNDV/M-F & CTGTGCTTGTGAAGGCGAGAGGT & 100 & KP742770 \\
\hline qNDV/M-R & TGGGGAGAGGCATTTGCTATAGGAT & & \\
\hline qGFP-F & CGACAAGCAGAAGAACGGCATCA & 154 & U55763 \\
\hline qGFP-R & GGACTGGGTGCTCAGGTAGTGGTT & & \\
\hline qGAPDH-F & TCAAGGCTGAGAACGGGAAACTTG & 117 & NM_204305 \\
\hline qGAPDH-R & TGGACTCCACAACATACTCAGCACC & & \\
\hline
\end{tabular}

at least three times, and the results are presented as the mean \pm standard deviation (SD). A $P$-value $<0.05$ was considered significant. P-values are indicated by asterisks $\left({ }^{*} P<0.05,{ }^{* *} P<0.01,{ }^{* * *} P<0.001\right)$.

\section{Results}

Interaction of the NDV M protein and chBRD2 protein in cellulo and in vitro

The chBRD2 protein is one of the cellular proteins found to interact with the NDV M protein using the yeast twohybrid screening system [11]. To further confirm their interaction, fluorescence co-localization, co-IP and pulldown assays were performed. As shown in Figure 1A, either EGFP-M and DsRed-chBRD2 fusion proteins or HA-M and Myc-chBRD2 fusion proteins showed clear co-localization in the nucleus in co-transfected DF-1 cells, demonstrating that the nuclear localization of the $M$ protein and chBRD2 protein was not affected by the fused tag. To verify the in cellulo interaction between the $M$ protein and chBRD2 protein, a co-IP assay with DF-1 cells transiently co-transfected with plasmids encoding HA-M and Myc-chBRD2 was carried out. Western blot analysis results showed that the fusion proteins HA-M and Myc-chBRD2 were normally expressed (Figure 1B). Moreover, the HA-M fusion protein but not HA tag in cell supernatants could be immunoprecipitated with Myc-chBRD2 when using an anti-Myc antibody (Figure $1 \mathrm{~B})$. In addition, the relatively pure recombinant proteins GST-M and His-chBRD2 were obtained after prokaryotic expression and protein purification (Figure $1 C$ ). Moreover, an in vitro binding assay of the fusion protein GST-M to the purified His-chBRD2 fusion protein showed that the His-chBRD2 protein was pulled down by the GST-M protein but not by the GST tag (Figure 1D) and that, in turn, the GST-M protein was also pulled down by the His-chBRD2 protein but not by the His tag (Figure 1E). These results demonstrated that the NDV $M$ protein physically interacted with the chBRD2 protein.

\section{Mapping the binding domains between the NDV M protein and chBRD2 protein}

The NDV $M$ protein is reported to contain three NESs and one NLS [12, 30], and the BRD2 protein contains one NLS, two bromodomains (BD1 and BD2), and one extra-terminal (ET) domain [24]. To determine the interaction regions between the $M$ protein and chBRD2 protein, a series of $M$ and chBRD2 truncation mutants were constructed to search for their binding domains using a co-IP assay (Figures 2A and B, upper panels). The binding experiments showed that the C-terminus (aa 264313 ) of the $M$ protein was essential for chBRD2 binding since HA-M(1-364), HA-M(264-364) and HA-M(264313) could be immunoprecipitated with Myc-chBRD2, while the other truncation or deletion mutants of the HA-tagged $M$ protein lost their binding activity to Myc-chBRD2 (Figure 2A, lower panel). In addition, only the fragments containing the ET domain (aa 619683) of chBRD2, such as Myc-chBRD2(1-779), MycchBRD2(511-779) and Myc-chBRD2(619-683), could interact with the HA-M protein (Figure $2 \mathrm{~B}$, lower panel). Therefore, these results suggested that the $\mathrm{C}$-terminus (aa 264-313) of the NDV M protein and the ET domain (aa 619-683) of the chBRD2 protein were responsible for interaction with each other.

\section{Conservation analysis of the BRD2/ET domain among humans and other species}

To understand the conservation of the BRD2/ET domain, the protein sequences of BRD2 from avian 
A

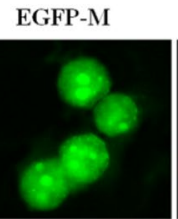

HA-M

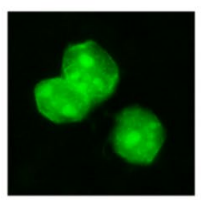

DsRed-chBRD2

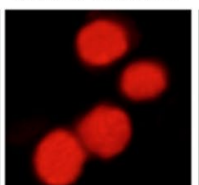

Myc-chBRD2

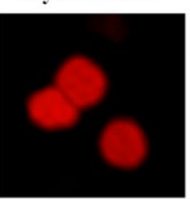

Merge

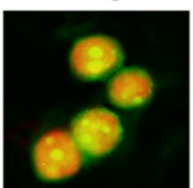

Merge

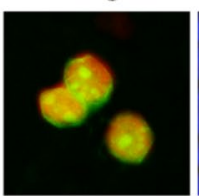

DAPI

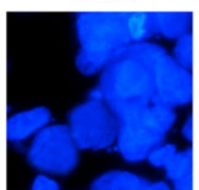

DAPI

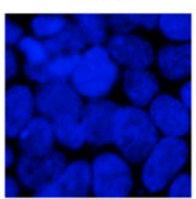

DAPI/Merge

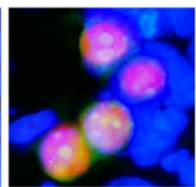

DAPI/Merge

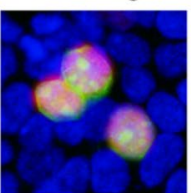

B

pCMV-HA-M

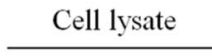

$\frac{\text { Co-IP: anti-Myc }}{+-\quad+}$

pCMV-HA

pCMV-Myc-chBRD2

pCMV-Myc

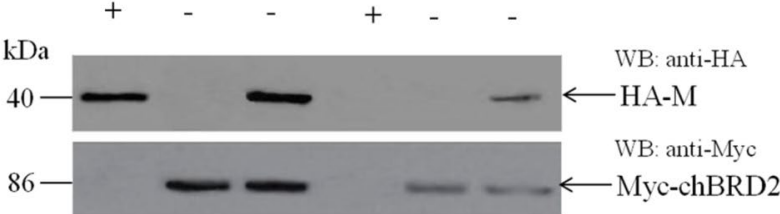

C

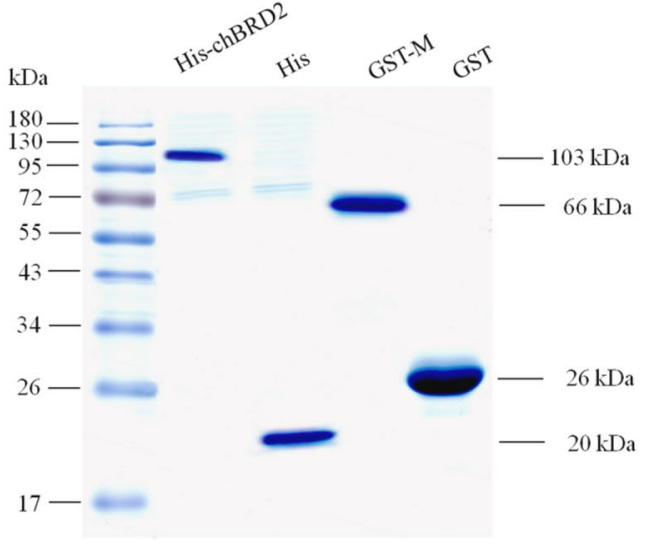

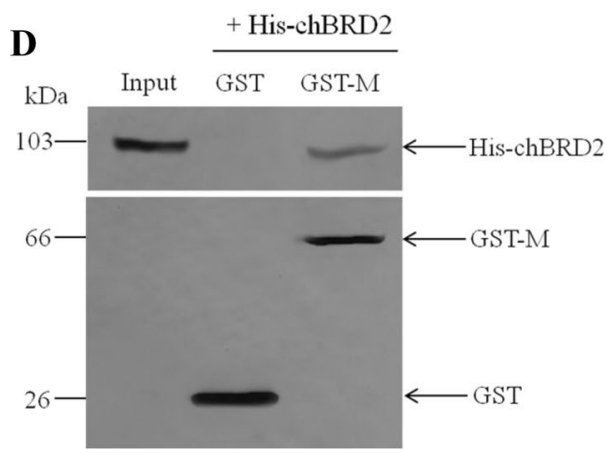

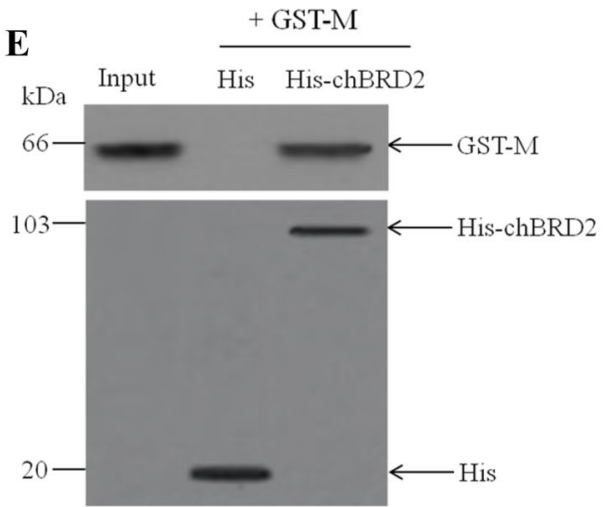

Figure 1 Identification of the interaction between the NDV M protein and chBRD2 protein. A Fluorescence co-localization of the NDV M protein and chBRD2 protein in plasmid-co-transfected cells. The plasmids pEGFP-M and pDsRed-chBRD2 or pCMV-HA-M and pCMV-Myc-chBRD2 were co-transfected into DF-1 cells. Twenty-four hours after transfection, direct fluorescence methods and indirect immunofluorescence assays were used to observe the fluorescence of EGFP-M and DsRed-chBRD2 or HA-M and Myc-chBRD2. DAPI was used to detect nuclei. The original magnification was $1 \times 200$. B Identification of the interaction between the M protein and chBRD2 protein by co-IP assay. The indicated plasmids were co-transfected into DF-1 cells, and reciprocal co-IP assays were performed to identify the interaction between HA-M and Myc-chBRD2 in DF-1 cells at 36 hpt. C A Coomassie stained gel showing the bacterial expression purified proteins. The His-tagged chBRD2 (His-chBRD2), His tag, GST-tagged M (GST-M), and GST tag were expressed in E. coli BL21 (DE3), and the soluble His-chBRD2 and His tag or GST-M and GST tag were purified on Ni-NTA His*Bind Resin or Glutathione-Sepharose 4B beads, respectively. The bacterial expression purified proteins were detected by SDS-PAGE along with Coomassie blue staining. Identification of the interaction between the M protein and chBRD2 protein by His pull-down assay (D) and GST pull-down assay (E). Upper panel, the purified His-chBRD2 or GST-M (Input) and the pull-downed His-chBRD2 or GST-M were detected by western blot using a mouse anti-His or anti-GST antibody. Lower panel, the bait proteins (GST and GST-M or His and His-chBRD2) in the pull-down fractions were detected by western blot using a mouse anti-GST or anti-His antibody. 


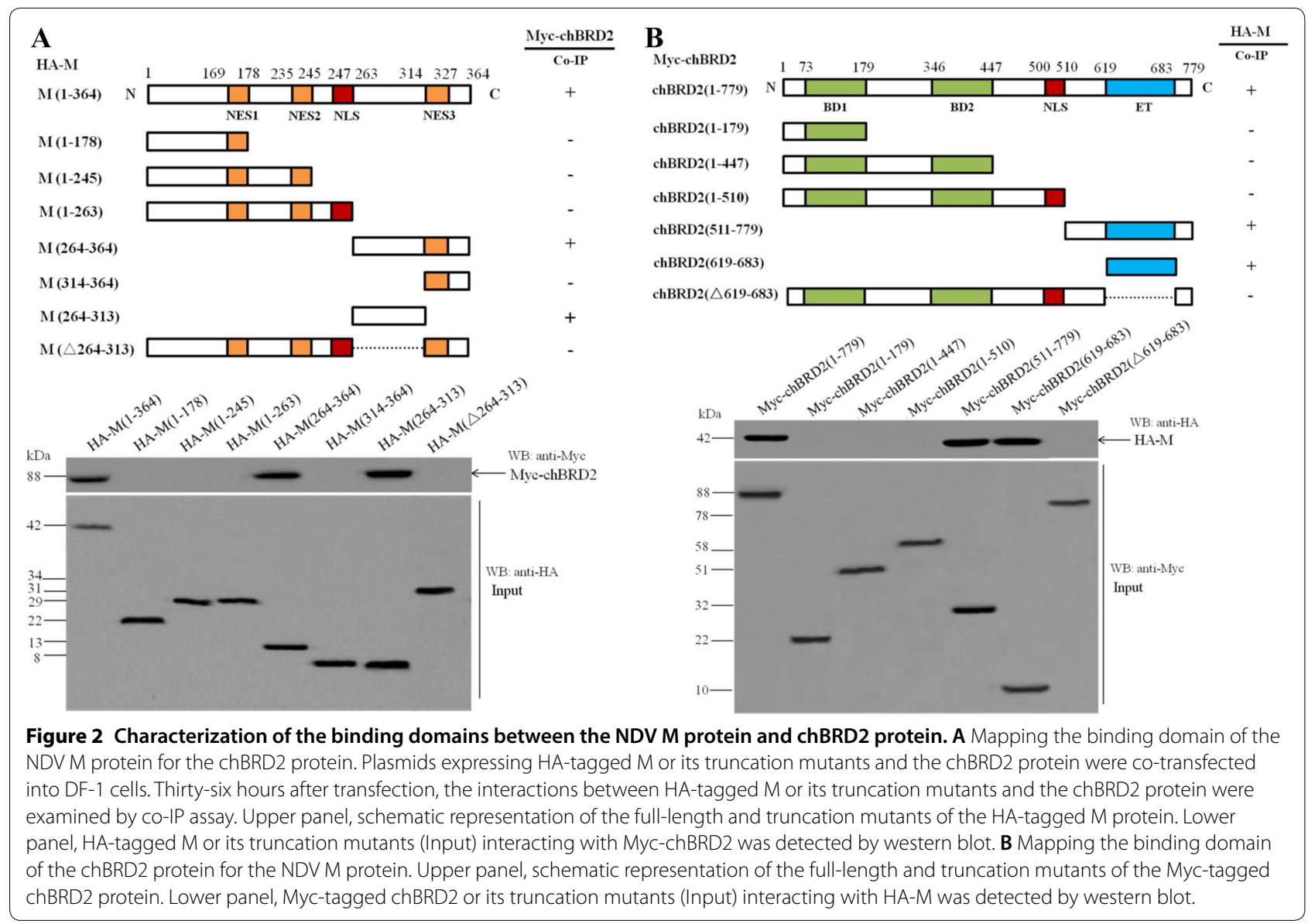

species (Gallus gallus, Chrysolophus pictus, and Nipponia nippon), humans and other mammals, including Pan paniscus, Rattus norvegicus, Mus musculus, Mesocricetus auratus, Sus scrofa, and Bos taurus, were analysed. The results of multiple sequence alignment showed that the amino acids of the BRD2/ET domain were absolutely conserved between humans and other mammals, but there were two amino acid changes (T621 and S649) found in the chBRD2/ET domain (Figure 3A). Further three-dimensional crystal structure modelling of huBRD2/ET and chBRD2/ET showed that the T621 and S649 changes in the chBRD2/ET domain mainly reduced $\alpha$-helix formation and obviously changed the molecular structure surface when compared to the huBRD2/ET domain (Figure 3B). However, it is surprising that the two different amino acid changes found in the BRD2/ET domain of humans and other mammals did not disrupt the BRD2-M interaction or the chBRD2-M interaction (Figure 3C). Thus, these data indicated that other regions or sites of the chBRD2/ET domain might contribute to interactions with the NDV M protein.

\section{The NDV M protein downregulates the transcription of the chBRD2 gene}

To determine whether the interaction of the NDV $M$ protein with the chBRD2 protein affects the transcription of chBRD2, the transcription levels of the chBRD2 gene were evaluated by qRT-PCR in virus-infected or plasmid-transfected DF-1 cells. As shown in Figure 4A, the expression levels of the NDV M protein gradually increased from $0.01 \mathrm{MOI}$ to $1 \mathrm{MOI}$ of NDV strain rSS$1 \mathrm{GFP}$ at 6,12 and $24 \mathrm{hpi}$. In contrast, the transcription levels of the $c h B R D 2$ gene were obviously decreased with increasing infective dose of rSS1GFP from 6 to 24 hpi (downregulated from 0.33 to 0.48 times in the 0.01 MOI group, from 0.39 to 0.62 times in the $0.01 \mathrm{MOI}$ group, from 0.60 to 0.87 times in the $1 \mathrm{MOI}$ group (Figure 4B). Next, different amounts of the plasmid pEGFP$\mathrm{M}$, pEGFP-M $(\Delta 264-313)$ or pEGFP-C1 $(1.0 \mu \mathrm{g}, 2.0 \mu \mathrm{g}$, or $4.0 \mu \mathrm{g}$ ) were transfected into DF-1 cells to examine its effect on the transcription of the $\operatorname{ch} B R D 2$ gene. The results showed that the fusion proteins EGFP-M and EGFP-M $(\Delta 264-313)$ and the EGFP tag more highly expressed when DF-1 cells were transfected with $4.0 \mu \mathrm{g}$ 


\begin{tabular}{|c|c|c|}
\hline \multirow{3}{*}{ A } & & * * \\
\hline & $\begin{array}{l}\text { Homo sapiens (AAH63840) } \\
\text { Paunus }\end{array}$ & PMSYDEKRQLSLDI NKLPGEKL GRVVHI I QAREPSLRDSNPEEI EI DFETLKPSTLRELERYVLS \\
\hline & $\begin{array}{l}\text { Pan paniscus (XP_024785040) } \\
\text { Rattusnorvegicus (NP_997660) }\end{array}$ & PMSYDEKRQLSLDI NKLPGEKL GRVVHI I QAREPSLRDSNPEEI EI DFETLKPSTLRELERYVLS \\
\hline \multirow{2}{*}{ BRD2 } & Musmusculus (NP 03+368) & $\begin{array}{l}\text { PMSYDEKRQLSLDI NKL PGEKL GRVVHI I QAREPSLRDSNPEEI EI DFETLKPSTLRELERYVLS } \\
\text { PMSYDEKRLSLDI NKLPGEKL GRVVHI GAREPSLRDSNPEEI EI DFETLKPSTLRELEYYLS }\end{array}$ \\
\hline & Mesocricetus auratus (XP_005086918) & PMSYDEKRQLSLDI NKLPGEKL GRVVHI I QAREPSLRDSNPEEI EI DFETLKPSTLRELERYVLS \\
\hline \multirow[t]{5}{*}{ ET domain } & Sus scrofa (XP_020953547) & PMSYDEKRQL SLDI NKLPGEKL GRVVHI I QAREPSLRDSNPEEI EI DFETLKPSTLRELERYVLS \\
\hline & Bosfaurus (XP_02+839204) & PMSYDEKRQLSLDI NKLPGEKL GRVVHI I QAREPSLRDSNPEEI EI DFETLKPSTLRELERYVLS \\
\hline & $\begin{array}{l}\text { Gallus gallus (XP_015150446) } \\
\text { Chrisoloplus pictus(AFN53616) }\end{array}$ & PMTYOEKRQLSLDI NKLPGEKL GRVVHI I QSREPSLRDSNPEEI EI DFETLKPSTLRELERYVLS \\
\hline & $\begin{array}{l}\text { Cryjsoloplinuspictus(AFNes616) } \\
\text { Nipponia nippon (ALP32491) }\end{array}$ & PMTYOEKRQLSLDI NKLPGEKL GRVVHI I OSREPSLRDSNPEEI EI DFETLKPSTLRELERYVLS \\
\hline & & PMTYDEKRQLSLDI NKLPGEKL GRVVHI I QSREPSLRDSNPEEI EI DFETLKPSTLRELERYVLS \\
\hline
\end{tabular}

B

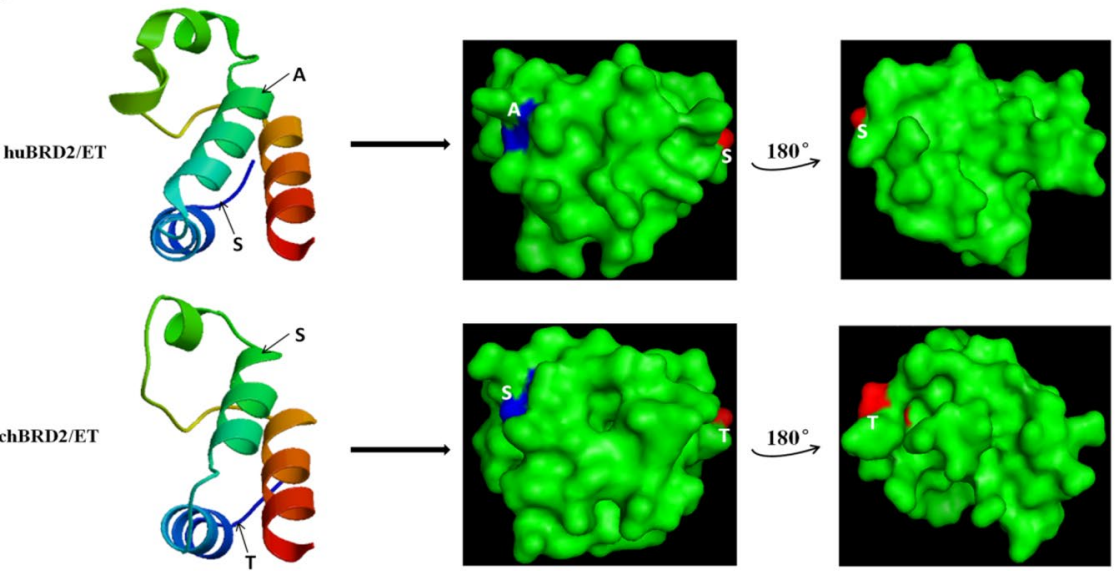

C

Gallus gallus chBRD2ET PMTYDEKRQLSLDINKLPGEKLGRVVHIQQSREPSLRDSNPEEIEIDFETLKPSTLRELERYVLS Homo sapiens $\mid$ huBRD2/ET PMSYDEKRQLSLDINKLPGEKLGRVVHIIQAREPSLRDSNPEEIEIDFETLKPSTLRELERYVLS

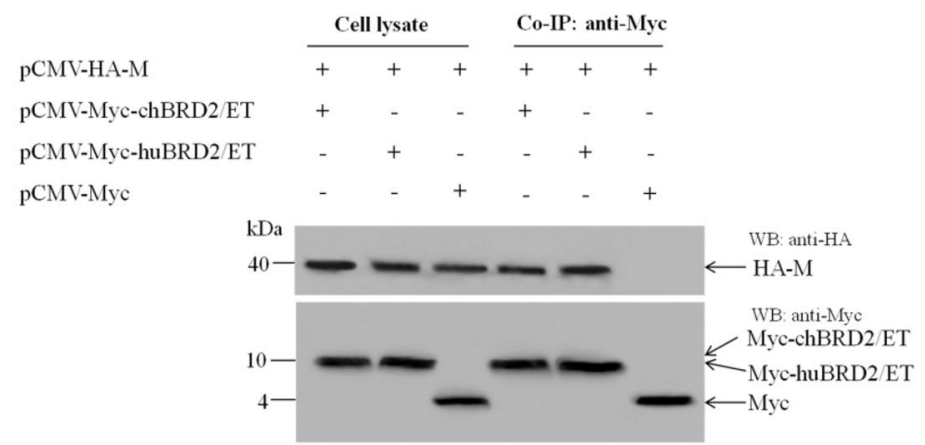

Figure 3 Conservation analysis of the BRD2/ET domain from different species. A The complete coding sequences of the BRD2 gene from humans and other selected species were retrieved from the GenBank database. The amino acid sequences of the BRD2 protein (huBRD2 as the template) were analysed using the Clustal W multiple alignment algorithm in the MegAlign program of the DNASTAR Lasergene package. The different amino acids were marked in boxes and asterisks. B The three-dimensional crystal structures of the huBRD2/ET domain and chBRD2/ ET domain (PDB accession no. 6CUI) were analysed using PyMOL. The different amino acids, including S in huBRD2/ET and T in chBRD2 and $\mathrm{A}$ in huBRD2/ET and $\mathrm{S}$ in chBRD2/ET, were marked in red and blue, respectively. C Identification of the interaction between the chBRD2/ET or huBRD2/ET domain and NDV M protein. Upper panel, schematic representation of the chBRD2/ET domain and huBRD2/ET domain. Lower panel, the combined plasmids were co-transfected into DF-1 cells, and $36 \mathrm{~h}$ after transfection, the interaction between HA-M and Myc-chBRD2/ET or Myc-huBRD2/ET was identified by co-IP assay.

of plasmid at $48 \mathrm{hpt}$ than after transfection with $1.0 \mu \mathrm{g}$ or $2.0 \mu \mathrm{g}$ of each plasmid (Figure $4 \mathrm{C}$ ). In addition, the transcription levels of the $\operatorname{chBRD} 2$ gene were relatively lower with transfection with $2.0 \mu \mathrm{g}$ or $4.0 \mu \mathrm{g}$ of pEGFP-M (downregulated 0.48 times and 0.66 times, respectively) than those of the pEGFP-M $(\Delta 264-313)$ group, pEGFP$\mathrm{C} 1$ group and non-transfected cells (Figure 4D). Therefore, these results suggested that the NDV $M$ protein could negatively regulate the transcription of the chBRD2 gene in a dose-dependent manner. 


\begin{abstract}
(See figure on next page.)
Figure 4 The effect of the NDV M protein on the transcription of the chBRD2 gene. A The expression levels of the NDV M protein in different infection doses of NDV-infected DF-1 cells. DF-1 cells were infected with rSS1GFP at an MOl of $0.01,0.1$ or 1 . The expression levels of the M protein in rSS1GFP-infected DF-1 cells were detected at 6, 12 and $24 \mathrm{hpi}$. The relative expression levels of the M protein compared to control GAPDH expression levels were determined by densitometry using ImageJ software version 1.8.0. Error bars represent standard deviations (mean \pm SD) $\left({ }^{*} P<0.05 ;{ }^{* *} P<0.01 ;{ }^{* *} P<0.001\right.$ compared to the value of the $0.01 \mathrm{MOI}$ group). B Transcription levels of the chBRD2 gene in DF-1 cells infected with different doses of NDV at 6, 12 and 24 hpi. The relative transcription levels of the chBRD2 gene were compared with those of the control GAPDH gene. Error bars represent standard deviations (mean \pm SD) $\left({ }^{*} P<0.05 ;{ }^{* *} P<0.01 ;{ }^{* * *} P<0.001\right.$ compared to the value of non-infected cells). C The expression levels of EGFP-M, EGFP-M( $\triangle 264-313)$ and EGFP in DF-1 cells transfected with different doses of plasmid. DF-1 cells were transfected with the plasmid pEGFP-M, pEGFP-M( $\triangle 264-313)$, or pEGFP-C1 at a dose of $1.0 \mu \mathrm{g}, 2.0 \mu \mathrm{g}$ or $4.0 \mu \mathrm{g}$. The expression levels of EGFP-M, EGFP-M $(\triangle 264-313)$ and EGFP in plasmid-transfected cells were examined at $36 \mathrm{hpt}$. The relative expression levels of the indicated proteins to control GAPDH expression levels were determined by densitometry using ImageJ software version 1.8.0. D Transcription levels of the chBRD2 gene in DF-1 cells transfected with different doses of pEGFP-M, pEGFP-M( $\triangle 264-313)$, or pEGFP-C1. The relative transcription levels of the chBRD2 gene were compared with those of the control GAPDH gene. Error bars represent standard deviations (mean \pm SD) $\left(^{* *} P<0.01\right.$; ${ }^{* * *} P<0.001$ compared to the value of non-transfected cells).
\end{abstract}

\section{Knockdown of chBRD2 promotes NDV replication in DF-1 cells}

To further investigate the role of chBRD2 in the replication of NDV, siRNA-mediated knockdown of chBRD2 in DF-1 cells infected with rSS1GFP was performed. Three pairs of chBRD2 siRNAs (Table 3) were synthesized and then transfected into DF-1 cells. The results of qRT-PCR analysis showed that chBRD2 siRNA\#2 could more effectively reduce the transcription level of the chBRD2 gene than other chBRD2 siRNAs and control siRNA (Figure $5 \mathrm{~A}$ ). The replication ability and cytopathogenicity of rSS1GFP in chBRD2 siRNA\#2- or control siRNA-treated DF-1 cells or non-transfected cells were then evaluated. The results of multicycle growth kinetic analysis revealed that the virus titres of rSS1GFP in chBRD2 siRNA\#2treated DF-1 cells were relatively higher (increased from 1.2 to 1.5 titre) than those in control siRNA-treated cells or non-transfected cells from 12 to $36 \mathrm{hpi}(P<0.01)$ (Figure $5 \mathrm{~B})$. In addition, the CPE caused by rSS1GFP infection in chBRD2 siRNA\#2-treated cells started at $12 \mathrm{hpi}$, and the cell monolayer began to be destroyed at $36 \mathrm{hpi}$, but the CPE in control siRNA-treated cells started to appear at $24 \mathrm{hpi}$, and cell monolayer destruction was not observed at 36 hpi (Figure 5C).

To examine whether the enhanced replication and cytopathogenicity of rSS1GFP was associated with increased viral RNA synthesis and transcription, chBRD2 siRNA\#2- or control siRNA-treated cells or non-transfected cells were infected with rSS1GFP, and the viral RNA levels and related gene transcription levels in rSS1GFP-infected cells were analysed by qRT-PCR. The results showed that the relative viral RNA levels (corresponding to the $N P$ and $P$ genes) between chBRD2 siRNA\#2- and control siRNA-treated cells or non-transfected cells had statistically significant differences at $12 \mathrm{hpi}(P<0.05)$, which continued to increase at $24 \mathrm{hpi}$ $(P<0.01)$ (Figure 5D). In addition, the relative viral gene transcription levels (corresponding to the $M$ and GFP genes) in chBRD2 siRNA\#2-treated cells were more increased than those in control siRNA-treated cells or non-transfected cells at 6 hpi $(P<0.05)$ and significantly higher at 12 and 24 hpi $(P<0.01)$ (Figure $5 \mathrm{E})$. Consistent with the relative transcription levels of the $M$ and GFP genes, the expression levels of the M and GFP proteins were also increased more in chBRD2 siRNA\#2-treated cells than in control siRNA-treated cells at different time points (Figure 5F). Together, these results indicated that knockdown of chBRD2 promoted the replication of NDV by upregulating viral RNA synthesis and transcription at early time points after infection.

\section{Overexpression of chBRD2 restricts NDV replication in DF-1 cells}

Because chBRD2 knockdown affected NDV replication, it was intriguing to further determine whether chBRD2 overexpression could also affect NDV replication. Therefore, DF-1 cells overexpressing DsRed or DsRed-chBRD2 were generated. The expression of DsRed or DsRed-chBRD2 in DF-1 cells was obviously observed by fluorescence observation (Figure 6A). The results of qRT-PCR analysis showed that the transcription level of the chBRD2 gene in pDsRed-chBRD2-transfected cells was much higher than that in pDsRed-C1-transfected cells or non-transfected cells $(P<0.001)$ (Figure 6B). In addition, overexpression of DsRed-chBRD2 reduced the virus titres (from 0.5 to 1.1 titre) of rSS1GFP from 24 to $72 \mathrm{hpi}$, which especially decreased the virus titres at 36 hpi $(P<0.001)$ (Figure $6 \mathrm{C})$. Then, the effect of chBRD2 overexpression on viral RNA synthesis and transcription was investigated. As shown in Figure 6D, the relative viral RNA levels (corresponding to the $N P$ and $P$ genes) in DsRed-chBRD2-overexpressing cells were more decreased at 12 and 24 hpi than those in DsRedoverexpressing cells or non-transfected cells. In addition, 

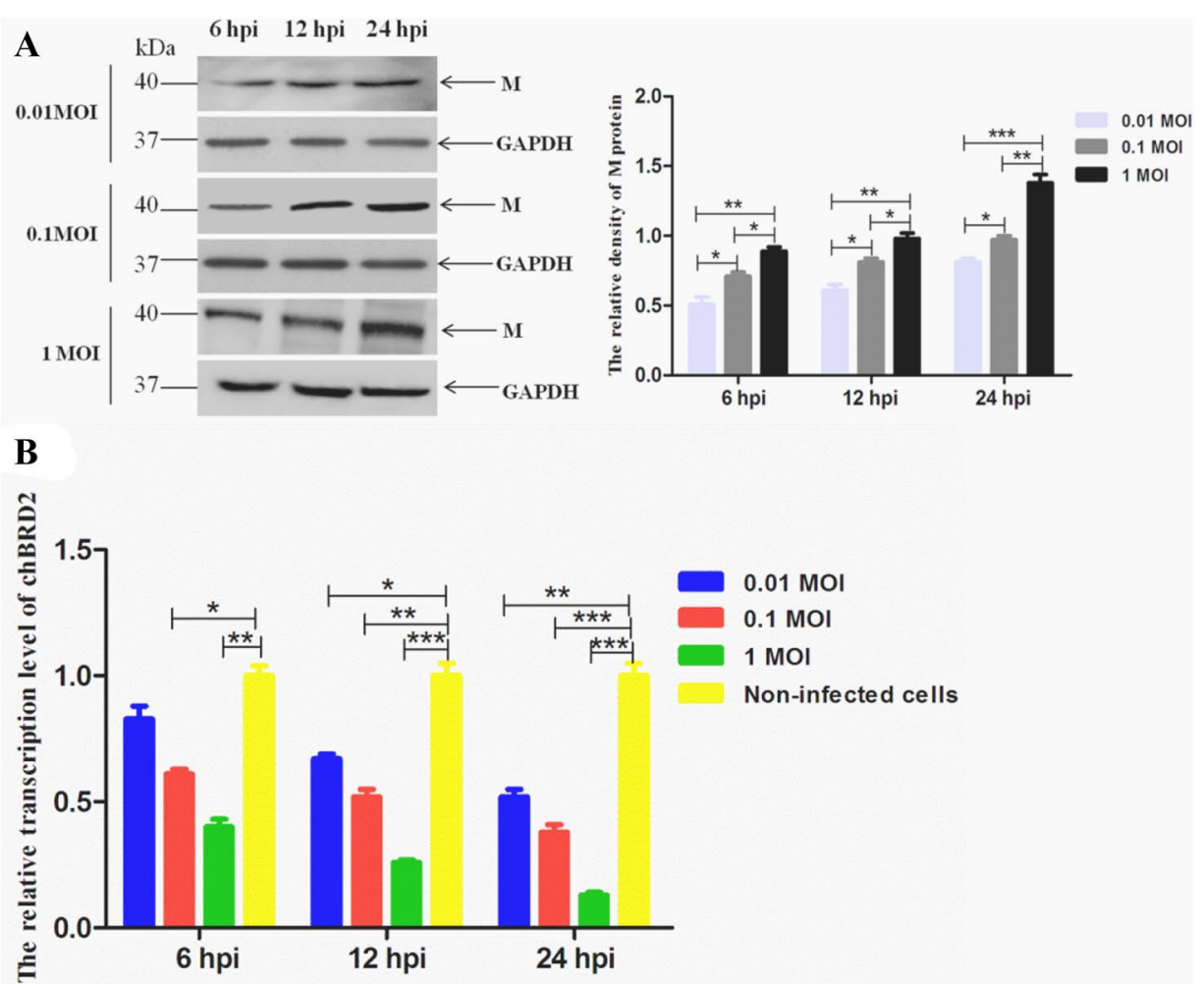

C
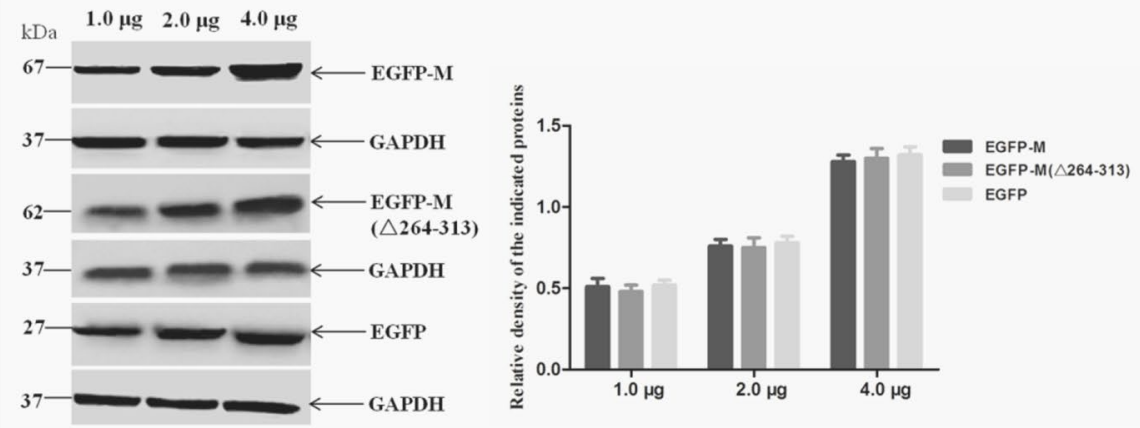

D

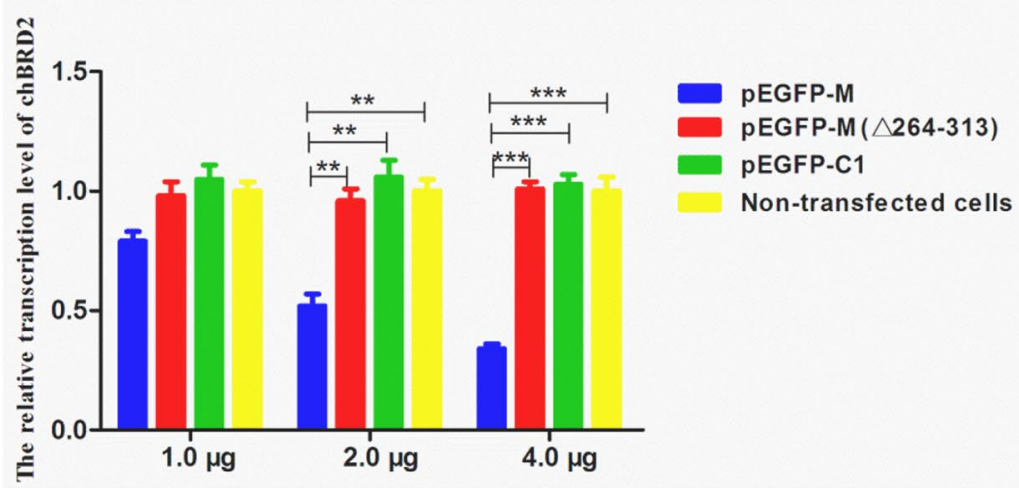




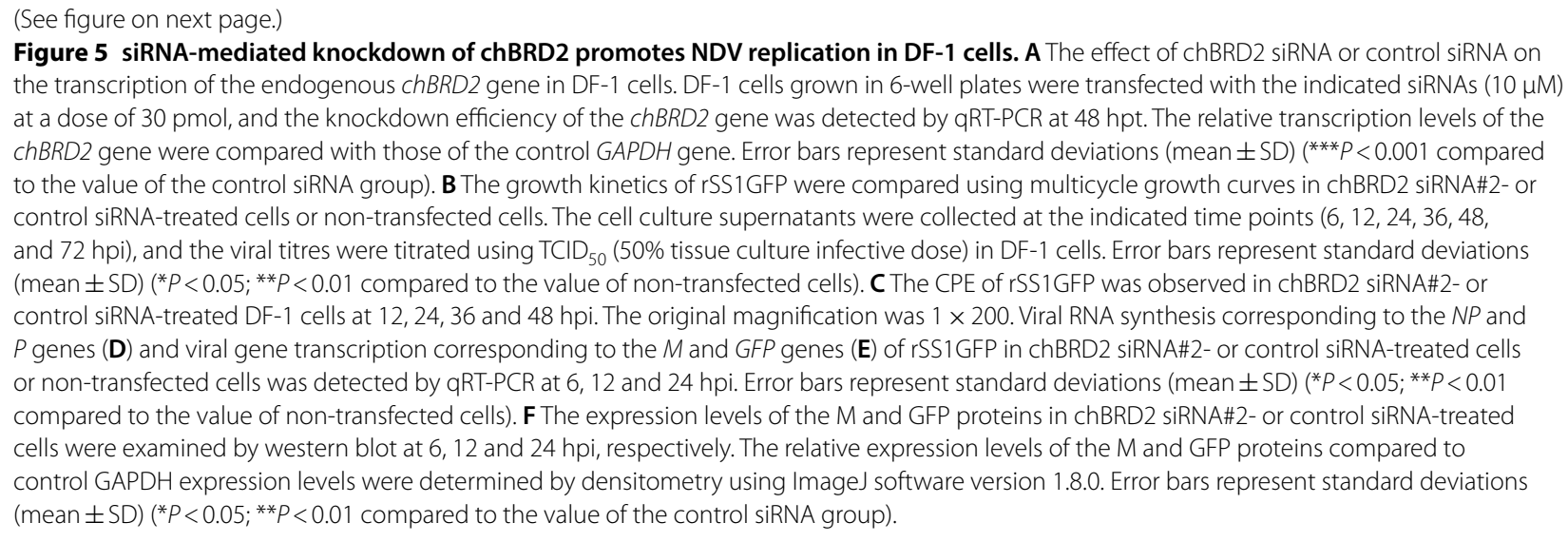

the relative transcription levels of the $M$ and GFP genes in DsRed-chBRD2-overexpressing cells were much lower than those in DsRed-overexpressing cells or non-transfected cells at 6 hpi $(P<0.05)$ and especially decreased (from 0.45 to 0.56 times) at 12 and $24 \mathrm{hpi}(P<0.01)$ (Figure $6 \mathrm{E})$. Meanwhile, the transcription expression levels of the $\mathrm{M}$ and GFP proteins were also obviously decreased (from 0.49 to 0.58 times) in DsRed-chBRD2-overexpressing cells in comparison to those in DsRed-overexpressing cells at 12 and 24 hpi (Figure 6F). Overall, these results suggested that overexpression of chBRD2 could reduce the replication of NDV by downregulating viral RNA synthesis and transcription.

\section{Discussion}

Studying virus-host protein-protein interactions is an important strategy to better understand the functions of viral proteins [31, 32]. Regarding paramyxovirus M protein and host protein interactions, it has been reported that the interaction of the Nipah and Hendra virus $M$ proteins with AP3B1 protein promotes virus-like particle (VLP) production [33]. In addition, the angiomotin-like 1 protein interacts with the parainfluenza virus 5 (PIV5) $M$ protein [34] and acts as a linker between the PIV5 $M$ and NEDD4 ubiquitin ligases [35], which reveals a novel host factor recruitment strategy for paramyxoviruses to achieve VLP production and virus budding. Moreover, a recent study showed that annexin A2 interacting with the measles virus $(\mathrm{MeV}) \mathrm{M}$ protein mediates the plasma membrane localization of the $\mathrm{M}$ protein and assists the assembly and budding of progeny virions [36]. All of these findings accelerate our understanding of the potential functions of paramyxovirus M proteins. For the NDV $\mathrm{M}$ protein, one familiar function is the core role of the $\mathrm{M}$ protein in the assembly and budding of NDV $[7,13]$, and another function is the hypothesized transcriptional inhibition of the M protein in the nucleus, which is analogous to the $\mathrm{M}$ proteins of human respiratory syncytial virus (HRSV) [37], vesicular stomatitis virus (VSV) [38] and $\mathrm{MeV}$ [39]. In recent years, although several studies have demonstrated that the interactions of the $M$ protein with host proteins are crucial for the replication and pathogenicity of NDV [11, 18-20], none of these host proteins are associated with nuclear proteins or the budding functions of the NDV M protein. Therefore, additional NDV $\mathrm{M}$-interacting host proteins remain to be further identified and studied.

In our previous study, the chBRD2 protein was identified as a new NDV M-interacting partner through yeast two-hybrid screening [11]. BRD2 is known as a nuclearlocalized serine-threonine kinase and plays pivotal roles in the transcriptional control of diverse genes [21, 23]. However, the role of the NDV M-chBRD2 interaction in the replication of NDV remains unclear. In this study, the interaction between the $\mathrm{M}$ protein and chBRD2 protein was first confirmed by fluorescence co-localization, co-IP and pull-down assays (Figure 1). In addition, we found that the C-terminus (aa 264-313) of the M protein and the ET domain (aa 619-683) of the chBRD2 protein were the binding domains (Figure 2). It has been shown that chBRD2 mainly contains two tandem BD domains and an ET domain. The BD domain is found to bind acetyl-lysine residues in histones and is required for the epigenetic regulation of gene transcription by interacting with nucleosomes within chromatin [24, 25], while the ET domain mainly performs its transcriptional regulatory function by recruiting specific effector proteins [26]. To date, viral proteins reported to interact with the BRD2/ET domain are the latency-associated nuclear antigen (LANA) of Kaposi sarcoma-associated herpesvirus (KSHV) [40] and the integrases of murine leukaemia virus (MLV) [41, 42] and porcine endogenous retrovirus [43]. Moreover, studies also found that amino acids, including R648, S651 and E685/E689, on the surface of the globular huBRD2/ET 


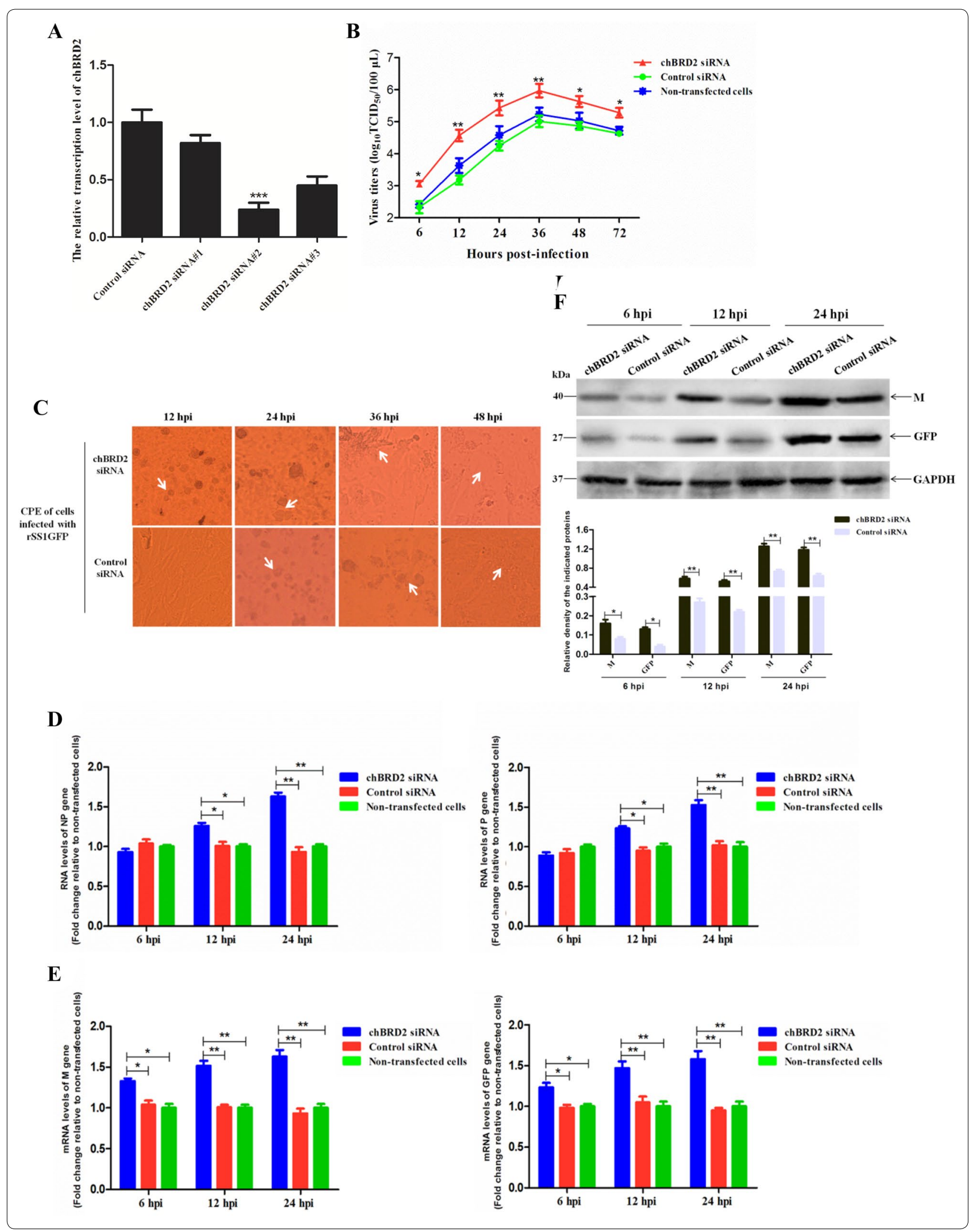




\begin{abstract}
(See figure on next page.)
Figure 6 Overexpression of chBRD2 reduces NDV replication in DF-1 cells. A Fluorescence observation of DsRed and DsRed-BRD2 in plasmid-transfected DF-1 cells. DF-1 cells were transfected with $3.0 \mathrm{\mu g}$ of pDsRed-C1 or pDsRed-chBRD2 to overexpress DsRed or DsRed-chBRD2. The fluorescence of DsRed and DsRed-chBRD2 was observed under an inverted fluorescence microscope at $36 \mathrm{hpt}$. DAPI was used to detect nuclei. The original magnification was $1 \times 100$. B The effect of DsRed or DsRed-BRD2 overexpression on the transcription of the chBRD2 gene in DF-1 cells. The transcription levels of the chBRD2 gene in pDsRed-C1- or pDsRed-chBRD2-transfected cells or non-transfected cells were detected by qRT-PCR at 36 hpt. The relative transcription levels of the chBRD2 gene were compared with those of the control GAPDH gene. Error bars represent standard deviations (mean \pm SD) $\left({ }^{* * *} P<0.001\right.$ compared to the value of non-transfected cells). C The growth kinetics of rSS1GFP were compared using multicycle growth curves in pDsRed-C1-or pDsRed-chBRD2-transfected cells or non-transfected cells. The cell culture supernatants were collected at the indicated time points $(6,12,24,36,48$, and $72 \mathrm{hpi})$, and the viral titres were titrated using $\mathrm{TCID}_{50}(50 \%$ tissue culture infective doses) in DF-1 cells. Error bars represent standard deviations (mean \pm SD) ${ }^{*} P<0.05 ;{ }^{*} P<0.01$; ${ }^{* * *} P<0.001$ compared to the value of non-transfected cells). Viral RNA synthesis corresponding to the NP and $P$ genes (D) and viral gene transcription corresponding to the $M$ and GFP genes (E) of rSS1GFP in pDsRed-C1- or pDsRed-chBRD2-transfected cells or non-transfected cells was detected by qRT-PCR at 6, 12 and 24 hpi. Error bars represent standard deviations (mean $\pm \mathrm{SD})\left({ }^{*} P<0.05 ;{ }^{* *} P<0.01 ;{ }^{* * *} P<0.001\right.$ compared to the value of non-transfected cells). $\mathbf{F}$ The expression levels of the $\mathrm{M}$ and GFP proteins in pDsRed-C1- or pDsRed-chBRD2-transfected cells or non-transfected cells were examined by western blot at 6, 12 and 24 hpi, respectively. The relative expression levels of the M and GFP proteins compared to control GAPDH expression levels were determined by densitometry using ImageJ software version 1.8.0. Error bars represent standard deviations (mean \pm SD) $\left(* P<0.05 ;{ }^{* *} P<0.01\right.$ compared to the value of non-transfected cells).
\end{abstract}

domain are responsible for the interaction with KSHV LANA [40]. Here, our findings showed that although two amino acids found in the chBRD2/ET domain were not similar to those in the BRD2/ET domain of humans and other mammals and changed the molecular structure surface of the chBRD2/ET domain (Figures 3A, B), they did not disrupt the BRD2-M interaction or the chBRD2$\mathrm{M}$ interaction (Figure 3C). It is important that the above amino acids (R648, S651 and E685/E689) in the globular huBRD2/ET domain are also conserved in avian species and other mammals (Figure 3A). Therefore, other regions or sites of the chBRD2/ET domain might be responsible for its interaction with the NDV M protein.

It has been demonstrated that $\mathrm{M}$ nuclear localization in some cytoplasmic RNA viruses, such as $\mathrm{MeV}$ and VSV, can inhibit host cell transcription independently of other viral components. For example, transiently overexpressed $\mathrm{MeV}$ M protein in plasmid-transfected cells binds to nuclear factors and inhibits in vitro transcription in a dose-dependent manner [39]. Other studies reported that the VSV M protein directly inhibits host cell transcription by inactivating host RNA polymerases I and II [44] and interacts with nuclear pore complexes to impair nuclear export of cellular mRNAs, which indirectly leads to a decrease and an increase in host cell and virus gene transcription, respectively [45-47]. Recently, we revealed that nuclear localization of the NDV M protein possibly affected host cell transcription because transcription repressor activity-related differentially expressed (DE) genes were upregulated and transcriptional activator activity-related DE genes were downregulated [10], and the expression of most DE proteins related to "transcription" and "RNA processing and modification" was significantly decreased in NDV-infected cells early in infection when compared to that in cells infected with the mutant
NDV harbouring M/NLS mutation [48]. More importantly, these upregulated or downregulated DE genes participated in regulating viral RNA synthesis and NDV replication [10]. In this study, we found that the transcription levels of the chBRD2 gene were obviously decreased in both virus-infected cells and pEGFP-M-transfected cells in a dose-dependent manner (Figure 4). Meanwhile, siRNA-mediated knockdown of chBRD2 promoted NDV replication by upregulating viral RNA synthesis and transcription (Figure 5), while overexpression of chBRD2 produced the opposite results in comparison to those of the chBRD2 knockdown experiment (Figure 6). Together, these findings suggested that downregulation of cellular transcription-related genes caused by $\mathrm{M}$ nuclear localization was beneficial for viral RNA synthesis and transcription to promote NDV replication.

BRD2 is a TATA-binding protein (TBP)-associated protein that recruits TBP into the E2F-1 transcriptional complex to perform transactivation effects [26]. In addition, a previous study showed that the major interaction subcomplexes of BRD2 include TBP-associated factors (TAFs), RNA Polymerase II, activated transcription factors, chromatin/histone modification enzymes and SWI/SNF remodelling complex components [49]. However, whether the NDV M-chBRD2 interaction reduces the expression of chBRD2-interacting proteins or chBRD2-involved transcription complexes and how this interaction regulates viral RNA synthesis and transcription must be determined. It is worth noting that the ET domains of BRD2, BRD3 and BRD4 are also very well conserved among human, mammalian and avian species [21, 24]. Thus, the chBRD3 and chBRD4 ET domains might also interact with the NDV M protein. In addition, some studies have reported that the interactions of the BRD2, BRD3, and BRD4 ET domains with 
A

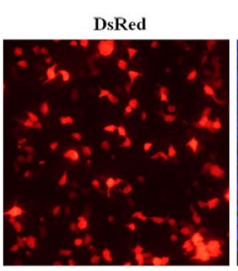

DsRed-BRD2
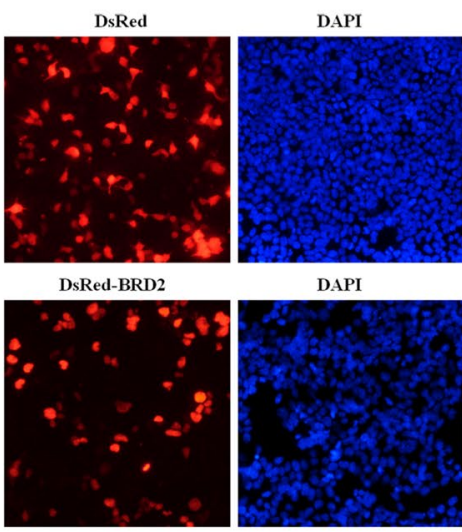

DAPI

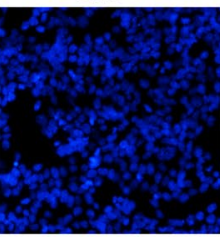

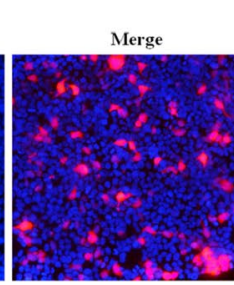

Merge

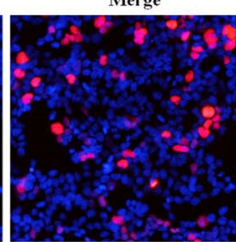

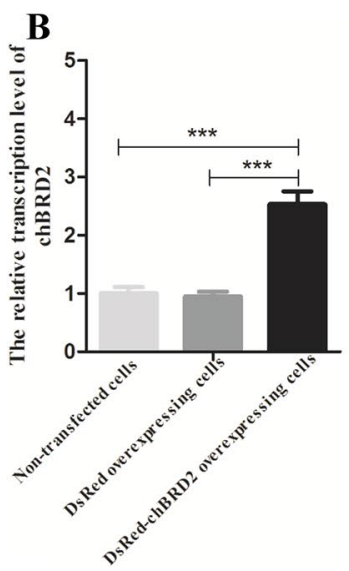
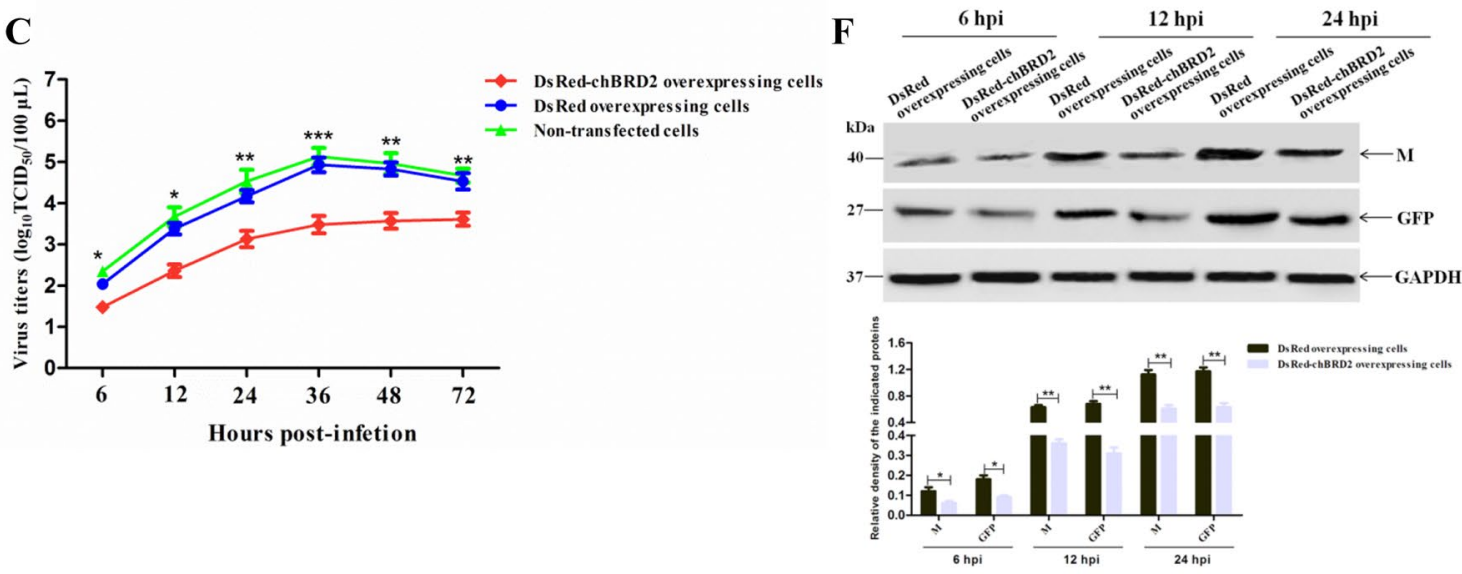

D
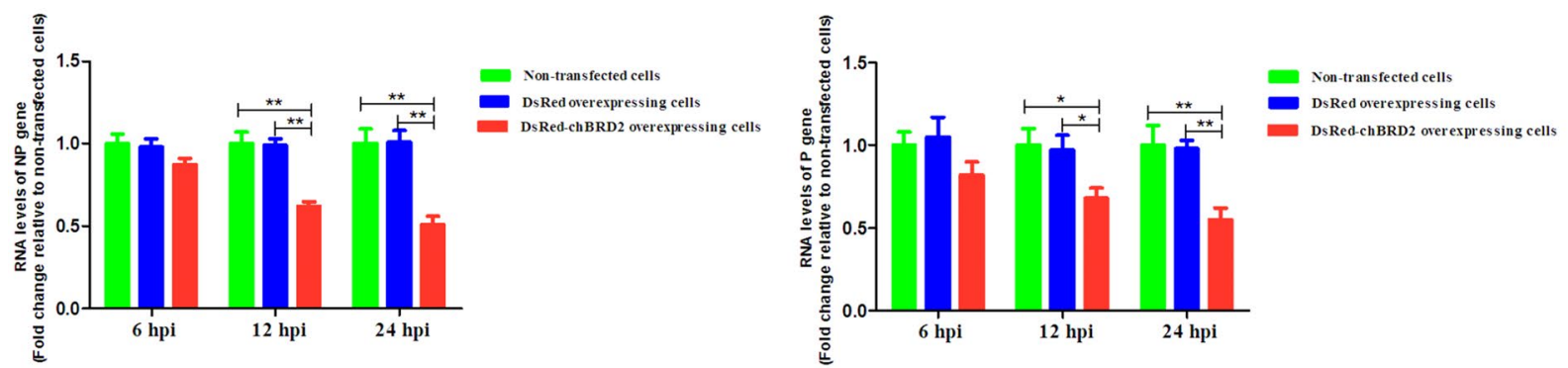

E
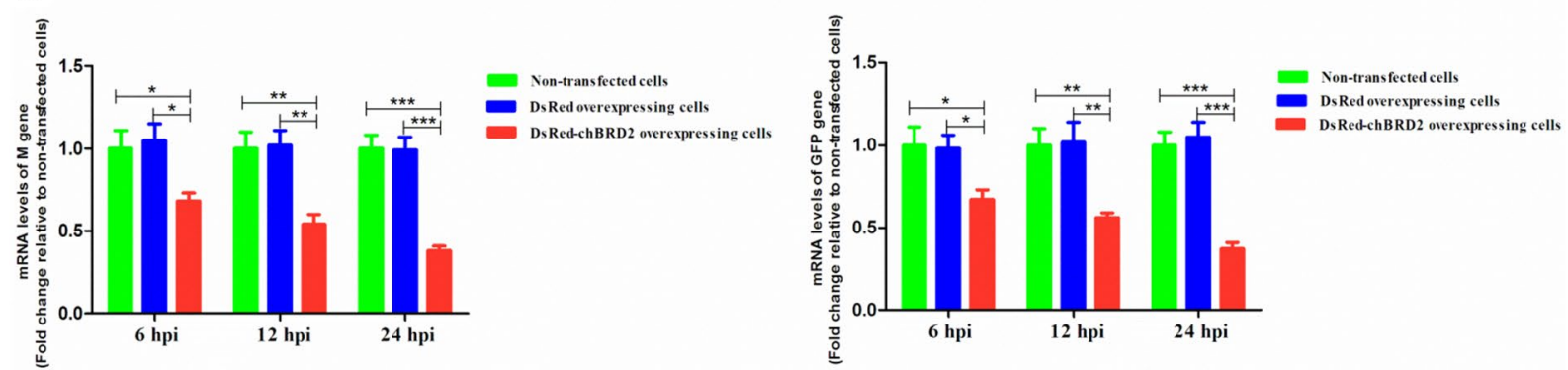
MLV integrase stimulate its catalytic activity and promote efficient MLV integration near active transcription units in the host genome $[41,50]$. Additionally, the ET domains of BRD2 and BRD4 binding on the surface of KSHV LANA (kLANA) are required for the formation of kLANA "nuclear speckles" and latent replication [40]. Unlike the functions of the BRD2/ET domain interacting with KSHV LANA and MLV integrase, NDV undergoes genomic replication, mRNA transcription, protein synthesis, and assembly of virus components in the cytoplasm, but the NDV M-chBRD2/ET interaction in the nucleus mainly assists in viral RNA synthesis and transcription until later in infection. Currently, accumulating studies have also revealed that the BRD2, BRD3 and BRD4 proteins participate in the host immune response against virus infection [51-53]. In our recent studies using quantitative proteomics analysis, we found that the parental virus rSS1GFP but not the mutant virus rSS1GFP-M/NLSm harbouring an NLS mutation in the $M$ protein reduced the expression of the BRD2, BRD3 and BRD4 proteins to different degrees in virus-infected cells, suggesting that the decreased expression of BRD2, BRD3 and BRD4 was caused by early nuclear localization of the $M$ protein [48]. Here, we also found that chBRD2 knockdown or overexpression continued to increase or reduce the viral titres in DF-1 cells, respectively, indicating that chBRD2 might be involved in the immune response against NDV infection. Therefore, further studies will be performed to identify the interactions between the $M$ protein and the chBRD3 and chBRD4 ET domains and to elucidate the role of chBRD2, chBRD3 and chBRD4 in the regulation of NDV replication in detail.

In conclusion, we demonstrated in this study that the NDV M protein interacted with the chBRD2 protein and downregulated its expression to facilitate viral RNA synthesis and transcription, which was beneficial for NDV replication. These findings will provide valuable information for better understanding the potential biological functions of M's nuclear localization in the NDV life cycle and aid in our understanding of the molecular mechanisms of NDV replication.

\footnotetext{
Abbreviations

BD: bromodomain; BET: bromodomain and extra-terminal domain; BRD2: bromodomain-containing protein 2; BRD3: bromodomain-containing protein 3; BRD4: bromodomain-containing protein 4; CO-IP: co-immunoprecipitation; CPE: cytopathic effect; ET: extra-terminal; hpi: hours post-infection; hpt: hours post-transfection; HRSV: human respiratory syncytial virus; KSHV: Kaposi sarcoma-associated herpesvirus; LANA: latency-associated nuclear antigen; MeV: measles virus; MLV: murine leukaemia virus; NDV: Newcastle disease virus; NES: nuclear export signal; NLS: nuclear localization signal; qRT-PCR: quantitative real-time PCR; siRNA: small interference RNA; $\mathrm{TCID}_{50}$ : 50\% tissue culture infective doses; VLP: virus-like particle; VSV: vesicular stomatitis virus.
}

\section{Acknowledgements}

The authors would like to thank the anonymous editors and reviewers for their valuable comments and suggestions that improved the quality of this manuscript.

\section{Authors' contributions}

ZQD conceived and designed these experiments and wrote the manuscript. YFH, LZ, CY, YBW and CQZ performed the experiments. HT and JQC performed the statistical analysis. All authors read and approved the final manuscript.

\section{Funding}

This work was supported by the National Natural Science Foundation of China (Grant Nos. 31960698 and 31760732), the Science and Technology Fund of Guizhou Province (Grant Nos. QKHJ-2020-1Y134), the Transformation and Industrialization of Scientific and Technological Achievements of Guizhou Province (Grant No. QKHKY-2017-055), and the Agricultural Research Project of Guizhou Province (Grant No. QKHZC-2016-2588).

\section{Availability of data and materials}

The datasets used and/or analysed during the current study are available from the corresponding author on reasonable request.

Ethics approval and consent to participate Not applicable.

\section{Consent to publish}

The details/images/videos will be freely available on the internet and may be seen by the general public.

\section{Competing interests}

The authors declare that they have no competing interests.

\section{Author details \\ ${ }^{1}$ Key Laboratory of Animal Genetics, Breeding and Reproduction in the Pla- teau Mountainous Region, Ministry of Education, Guizhou University, Guiyang, China. ${ }^{2}$ College of Animal Science, Guizhou University, Jiaxiu South Road, Huaxi District, Guiyang 550025, Guizhou, China.}

Received: 15 April 2020 Accepted: 10 September 2020

Published online: 22 September 2020

\section{References}

1. Dortmans JCFM, Koch G, Rottier PJM, Peeters BPH (2011) Virulence of Newcastle disease virus: what is known so far? Vet Res 42:122

2. Amarasinghe GK, Ayllón MA, Bào Y, Basler CF, Bavari S, Blasdell KR, Briese T, Brown PA, Bukreyev A, Balkema-Buschmann A, Buchholz UJ, Chabi-Jesus C, Chandran K, Chiapponi C, Crozier I, de Swart RL, Dietzgen RG, Dolnik O, Drexler JF, Dürrwald R, Dundon WG, Duprex WP, Dye JM, Easton AJ, Fooks AR, Formenty PBH, Fouchier RAM, Freitas-Astúa J, Griffiths A, Hewson R, Horie M, Hyndman TH, Jiāng D, Kitajima EW, Kobinger GP, Kondō H, Kurath G, Kuzmin IV, Lamb RA, Lavazza A, Lee B, Lelli D, Leroy EM, Lǐ J, Maes P, Marzano SL, Moreno A, Mühlberger E, Netesov SV, Nowotny N, Nylund A, Økland AL, Palacios G, Pályi B, Pawęska JT, Payne SL, Prosperi A, Ramos-González PL, Rima BK, Rota P, Rubbenstroth D, Shī M, Simmonds P, Smither SJ, Sozzi E, Spann K, Stenglein MD, Stone DM, Takada A, Tesh RB, Tomonaga K, Tordo N, Towner JS, van den Hoogen B, Vasilakis N, WahI V, Walker PJ, Wang LF, Whitfield AE, Williams JV, Zerbini FM, Zhāng T, Zhang YZ, Kuhn JH (2019) Taxonomy of the order Mononegavirales: update 2019. Arch Virol 164:1967-1980

3. de Leeuw OS, Peeters BP (1999) Complete nucleotide sequence of Newcastle disease virus: evidence for the existence of a new genus within the subfamily Paramyxovirinae. J Gen Virol 80:131-136

4. Peeters BP, Gruijthuijsen YK, de Leeuw OS, Gielkens AL (2000) Genome replication of Newcastle disease virus: involvement of the rule-of-six. Arch Virol 145:1829-1845

5. Battisti AJ, Meng G, Winkler DC, McGinnes LW, Plevka P, Steven AC, Morrison TG, Rossmann MG (2012) Structure and assembly of a paramyxovirus matrix protein. Proc Natl Acad Sci USA 109:13996-14000 
6. Harrison MS, Sakaguchi T, Schmitt AP (2010) Paramyxovirus assembly and budding: building particles that transmit infections. Int J Biochem Cell Bio 42:1416-1429

7. Pantua HD, McGinnes LW, Peeples ME, Morrison TG (2006) Requirements for the assembly and release of Newcastle disease virus-like particles. J Virol 80:11062-11073

8. Peeples ME, Wang C, Gupta KC, Coleman N (1992) Nuclear entry and nucleolar localization of the Newcastle disease virus (NDV) matrix protein occur early in infection and do not require other NDV proteins. J Virol 66:3263-3269

9. Duan Z, Li Q, He L, Zhao G, Chen J, Hu SL, Liu XF (2013) Application of green fluorescent protein-labeled assay for the study of subcellular localization of Newcastle disease virus matrix protein. JVirol Methods 194:118-122

10. Duan ZQ, Deng SS, Ji XQ, Zhao JF, Yuan C, Gao HB (2019) Nuclear localization of Newcastle disease virus matrix protein promotes virus replication by affecting viral RNA synthesis and transcription and inhibiting host cell transcription. Vet Res 50:22

11. Duan ZQ, Xu HX, Ji XQ, Zhao JF, Xu HQ, Hu Y, Deng SS, Hu SL, Liu XF (2018) Importin a5 negatively regulates importin $\beta 1$-mediated nuclear import of Newcastle disease virus matrix protein and viral replication and pathogenicity in chicken fibroblasts. Virulence 9:783-803

12. Duan ZQ, Song QQ, Wang YY, He L, Chen J, Zhu YM, Hu SL, Liu XF (2013) Characterization of signal sequences determining the nuclear export of Newcastle disease virus matrix protein. Arch Virol 158:2589-2595

13. Duan ZQ, Hu ZL, Zhu J, Xu HX, Chen J, Liu HM, Hu SL, Liu XF (2014) Mutations in the FPIV motif of Newcastle disease virus matrix protein attenuate virus replication and reduce virus budding. Arch Virol 159:1813-1819

14. Xu HX, Song QQ, Zhu J, Liu JJ, Cheng X, Hu SL, Wu S, Wang XQ, Liu XW, Liu XF (2016) A single R36Q mutation in the matrix protein of pigeon paramyxovirus type 1 reduces virus replication and shedding in pigeons. Arch Virol 161:1949-1955

15. Duan Z, Li J, Zhu J, Chen J, Xu HX, Wang YY, Liu HM, Hui SL, Liu XF (2014) A single amino acid mutation, R42A, in the Newcastle disease virus matrix protein abrogates its nuclear localization and attenuates viral replication and pathogenicity. J Gen Virol 95:1067-1073

16. Xu HX, Duan ZQ, Chen Y, Liu JJ, Cheng X, Liu JJ, Zhu J, Wang XQ, Liu XW, Hu SL, Liu XF (2016) Simultaneous mutation of G275A and P276A in the matrix protein of Newcastle disease virus decreases virus replication and budding. Arch Virol 161:3527-3533

17. Molouki A, Hsu YT, Jahanshiri F, Abdullah S, Rosli R, Yusoff K (2011) The matrix (M) protein of Newcastle disease virus binds to human Bax through its $\mathrm{BH} 3$ domain. Virol $\mathrm{J}$ 8:385

18. Li X, Li XQ, Cao H, Wang YQ, Zheng SJ (2013) Engagement of new castle disease virus (NDV) matrix (M) protein with charged multivesicular body protein (CHMP) 4 facilitates viral replication. Virus Res 171:80-88

19. Duan ZQ, Chen J, Xu HX, Zhu J, Li QH, He L, Liu HM, Hu SL, Liu XF (2014) The nucleolar phosphoprotein B23 targets Newcastle disease virus matrix protein to the nucleoli and facilitates viral replication. Virology 452:212-222

20. Shah M, Bharadwaj MSK, Gupta A, Kumar R, Kumar S (2019) Chicken viperin inhibits Newcastle disease virus infection in vitro: a possible interaction with the viral matrix protein. Cytokine 120:28-40

21. Zeng L, Zhou MM (2002) Bromodomain: an acetyl-lysine binding domain. FEBS Lett 513:124-128

22. Wu SY, Chiang CM (2007) The double bromodomain-containing chromatin adaptor Brd4 and transcriptional regulation. J Biol Chem 282:13141-13145

23. Huang H, Zhang J, Shen W, Wang X, Wu J, Wu J, Shi Y (2007) Solution structure of the second bromodomain of $\mathrm{Brd} 2$ and its specific interaction with acetylated histone tails. BMC Struct Biol 7:57

24. Taniguchi $Y$ (2016) The bromodomain and extra-terminal domain (BET) family: functional anatomy of BET paralogous proteins. Int J Mol Sci 17:1849

25. Tang LL, Nogales E, Ciferri C (2010) Structure and function of SWI/SNF chromatin remodeling complexes and mechanistic implications for transcription. Prog Biophys Mol Bio 102:122-128

26. Peng J, Dong W, Chen L, Zou T, Qi Y, Liu Y (2007) Brd2 is a TBP-associated protein and recruits TBP into E2F-1 transcriptional complex in response to serum stimulation. Mol Cell Biochem 294:45-54
27. Reed $\sqcup$, Muench $H$ (1938) A simple method of estimating fifty percent endpoints. Am J Epidemiol 27:493-497

28. Whelan SPJ, Wertz GW (2002) Transcription and replication initiate at separate sites on the vesicular stomatitis virus genome. Proc Natl Acad Sci USA 99:9178-9183

29. Yu XH, Cheng JL, He ZR, Li C, Song Y, Xue J, Yang HM, Zhang R, Zhang GZ (2017) The glutamic residue at position 402 in the C-terminus of Newcastle disease virus nucleoprotein is critical for the virus. Sci Rep 7:17471

30. Coleman NA, Peeples ME (1993) The matrix protein of Newcastle disease virus localizes to the nucleus via a bipartite nuclear localization signal. Virology 195:596-607

31. Brito AF, Pinney JW (2017) Protein-protein interactions in virus-host systems. Front Microbiol 8:1557

32. Goodacre N, Devkota P, Bae E, Wuchty S, Uetz P (2020) Protein-protein interactions of human viruses. Semin Cell Dev Biol 99:31-39

33. Sun WN, McCrory TS, Khaw WY, Petzing S, Myers T, Schmitt AP (2014) Matrix proteins of Nipah and Hendra viruses interact with beta subunits of AP-3 complexes. J Virol 88:13099-13110

34. Pei ZF, Bai YT, Schmitt AP (2010) PIV5 M protein interaction with host protein angiomotin-like 1. Virology 397:155-166

35. Ray G, Schmitt PT, Schmitt AP (2019) Angiomotin-like 1 links paramyxovirus $M$ proteins to NEDD4 family ubiquitin ligases. Viruses 11:128

36. Koga R, Kubota M, Hashiguchi T, Yanagi Y, Ohno S (2018) Annexin A2 mediates the localization of Measles virus matrix protein at the plasma membrane. J Virol 92:e00181-e00218

37. Ghildyal R, Baulch-Brown C, Mills J, Meanger J (2003) The matrix protein of human respiratory syncytial virus localises to the nucleus of infected cells and inhibits transcription. Arch Virol 148:1419-1429

38. Rajani KR, Kneller ELP, McKenzie MO, Horita DA, Chou JW, Lyles DS (2012) Complexes of vesicular stomatitis virus matrix protein with host Rae1 and Nup98 involved in inhibition of host transcription. PLoS Pathog 8:e1002929

39. Yu XL, Shahriari S, Li HM, Ghildyal R (2016) Measles virus matrix protein inhibits host cell transcription. PLoS One 11:e0161360

40. Hellert J, Weidner-Glunde M, Krausze J, Richter U, Adler H, Fedorov R, Pietrek M, Ruckert J, Ritter C, Schulz TF, Luhrs T (2013) A structural basis for BRD2/4-mediated host Chromatin interaction and oligomer assembly of Kaposi sarcoma-associated herpesvirus and murine gammaherpesvirus LANA proteins. PLoS Pathog 9:e1003640

41. De Rijck J, de Kogel C, Demeulemeester J, Vets S, El Ashkar S, Malani N, Bushman FD, Landuyt B, Husson SJ, Busschots K, Gijsbers R, Debyser Z (2013) The BET family of proteins targets Moloney murine leukemia virus integration near transcription start site. Cell Rep 5:886-894

42. Aiyer S, Swapna GVT, Malani N, Aramini JM, Schneider WM, Plumb MR, Ghanem M, Larue RC, Sharma A, Studamire B, Kvaratskhelia M, Bushman FD, Montelione GT, Roth MJ (2014) Altering murine leukemia virus integration through disruption of the integrase and BET protein family interaction. Nucleic Acids Res 42:5917-5928

43. Gallay K, Blot G, Chahpazoff M, Yajjou-Hamalian H, Confort MP, De Boisseson C, Leroux A, Luengo C, Fiorini F, Layigne M, Chebloune Y, Gouet P, Moreau K, Blanchard Y, Ronfort C (2019) In vitro, in cellulo and structura characterizations of the interaction between the integrase of Porcine Endogenous Retrovirus A/C and proteins of the BET family. Virology 532:69-81

44. Ahmed M, Lyles DS (1998) Effect of vesicular stomatitis virus matrix protein on transcription directed by host RNA polymerases I, II, and III. J Virol 72:8413-8419

45. Pan W, Song DG, He WQ, Lu HJ, Lan YG, Tong JZ, Gao F, Zhao K (2017) The matrix protein of vesicular stomatitis virus inhibits host-directed transcription of target genes via interaction with the TFIIH subunit p8. Vet Microbiol 208:82-88

46. von Kobbe C, van Deursen JM, Rodrigues JP, Sitterlin D, Bachi A, Wu X, Wilm M, Carmo-Fonseca M, Izaurralde E (2000) Vesicular stomatitis virus matrix protein inhibits host cell gene expression by targeting the nucleoporin Nup98. Mol Cell 6:1243-1452

47. Faria PA, Chakraborty P, Levay A, Barber GN, Ezelle HJ, Enninga J, Arana C, van Deursen J, Fontoura BM (2005) VSV disrupts the Rae1/mrnp41 mRNA nuclear export pathway. Mol Cell 17:93-102

48. Duan ZQ, Yuan C, Han YF, Zhou L, Zhao JF, Ruan Y, Chen JQ, Ni MM, Ji XQ (2020) TMT-based quantitative proteomics analysis reveals the attenuated 
replication mechanism of Newcastle disease virus caused by nuclear localization signal mutation in viral matrix protein. Virulence 11:607-635

49. Denis GV, McComb ME, Faller DV, Sinha A, Romesser PB, Costello CE (2006) Identification of transcription complexes that contain the double bromodomain protein $\mathrm{Brd} 2$ and chromatin remodeling machines. J Proteome Res 5:502-511

50. Gupta SS, Maetzig T, Maertens GN, Sharif A, Rothe M, Weidner-Glunde M, Galla M, Schambach A, Cherepanov P, Schulz TF (2013) Bromo- and extraterminal domain chromatin regulators serve as cofactors for murine leukemia virus integration. J Virol 87:12721-12736

51. Au-Yeung N, Horvath CM (2018) Histone H2A.Z suppression of interferonstimulated transcription and antiviral immunity is modulated by GCN5 and BRD2. iscience 6:68-82
52. Ren W, Wang C, Wang Q, Zhao D, Zhao K, Sun D, Liu X, Han C, Hou J, Li X, Zhang Q, Cao X, Li N (2017) Bromodomain protein Brd3 promotes Ifnb1 transcription via enhancing IRF3/p300 complex formation and recruitment to Ifnb1 promoter in macrophages. Sci Rep 7:39986

53. Wang J, Li GL, Ming SL, Wang CF, Shi LJ, Su BQ, Wu HT, Zeng L, Han YQ, Liu ZH, Jiang DW, Du YK, Li XD, Zhang GP, Yang GY, Chu BB (2020) BRD4 inhibition exerts anti-viral activity through DNA damage-dependent innate immune responses. PLoS Pathog 16:e1008429

\section{Publisher's Note}

Springer Nature remains neutral with regard to jurisdictional claims in published maps and institutional affiliations.
Ready to submit your research? Choose BMC and benefit from:

- fast, convenient online submission

- thorough peer review by experienced researchers in your field

- rapid publication on acceptance

- support for research data, including large and complex data types

- gold Open Access which fosters wider collaboration and increased citations

- maximum visibility for your research: over $100 \mathrm{M}$ website views per year

At BMC, research is always in progress.

Learn more biomedcentral.com/submissions 